\title{
New sets of eigenvalues in inverse scattering for inhomogeneous media and their determination from scattering data
}

\author{
Lorenzo Audibert ${ }^{1}$, Fioralba Cakoni ${ }^{2}$, Houssem Haddar ${ }^{3}$ \\ ${ }^{1}$ Departement PRISME, EDF R\&D, 6 quai Watier BP 49 Chatou, 78401 Cedex, \\ France \\ ${ }^{2}$ Department of Mathematics, Rutgers University, Piscataway, NJ 08854, USA \\ ${ }^{3}$ INRIA, CMAP, Ecole polytechnique, Université Paris Saclay, Route de Saclay, \\ 91128 Palaiseau, France \\ E-mail: lorenzo.audibert@edf.fr, fc292@math.rutgers.edu, \\ houssem.haddar@polytechnique.edu
}

\begin{abstract}
In this paper we develop a general mathematical framework to determine interior eigenvalues from a knowledge of the modified far field operator associated with an unknown (anisotropic) inhomogeneity. The modified far field operator is obtained by subtracting from the measured far field operator the computed far field operator corresponding to a well-posed scattering problem depending on one (possibly complex) parameter. Injectivity of this modified far field operator is related to an appropriate eigenvalue problem whose eigenvalues can be determined from the scattering data, and thus can be used to obtain information about material properties of the unknown inhomogeneity. We discuss here two examples of such modification leading to a Steklov eigenvalue problem, and a new type of the transmission eigenvalue problem. We present some numerical examples demonstrating the viability of our method for determining the interior eigenvalues form far field data.
\end{abstract}

Keywords: inverse scattering, inhomogenous media, generalized linear sampling method, Steklov eigenvalues, transmission eigenvalues.

AMS subject classifications: 35R30, 35J25, 35P25, 35P05 


\section{Introduction}

Spectral properties of operators associated with scattering problems provide essential information about scattering objects. However, the main question is whether such spectral features can be seen in the scattering data. As an example, the resonances (or scattering poles) constitute a fundamental part of scattering theory and their study has led to beautiful mathematics and has shed light into deeper understanding of direct and inverse scattering phenomena [21], [23]. But because the resonances are complex, it is difficult to determine them from scattering data unless they are near the real axis, which limits their use in inverse scattering. Hence now the question becomes, whether there are other sets of eigenvalues associated with the scattering problem which can be determined from corresponding scattering data. To be more specific, let us first introduce the scattering problem we consider here.

Suppose $D$ is a bounded domain in $\mathbb{R}^{m}, m=2,3$, with a piecewise smooth boundary $\partial D$ and having connected complement. The forward scattering problem we shall consider corresponds to the scattering by an anisotropic inhomogeneity supported in $D$ for acoustic waves $(m=3)$ or specially polarized electromagnetic waves $(m=2)$. In this case, the total field $u$ and the scattered field $u^{s}$ satisfy

$$
\begin{array}{ll}
\nabla \cdot A \nabla u+k^{2} n u=0 & \text { in } D \\
\Delta u^{s}+k^{2} u^{s}=0 & \text { in } \mathbb{R}^{m} \backslash \bar{D} \\
u-u^{s}=u^{i} & \text { on } \partial D \\
\frac{\partial u}{\partial \nu_{A}}-\frac{\partial u^{s}}{\partial \nu}=\frac{\partial u^{i}}{\partial \nu} & \text { on } \partial D \\
\lim _{r \rightarrow \infty} r^{\frac{m-1}{2}}\left(\frac{\partial u^{s}}{\partial r}-i k u^{s}\right)=0 &
\end{array}
$$

where $\frac{\partial u}{\partial \nu_{A}}:=\nu \cdot A \nabla u$, the incident field $u^{i}:=e^{i k x \cdot d}$ is a plane wave and the Sommerfeld radiation condition is satisfied uniformly with respect to $\hat{x}:=x /|x|, r=|x|$. Here $k>0$ is the wave number proportional to the interrogating frequency, $A$ is a $m \times m$ symmetric matrix with $L^{\infty}(D)$-entries such that

$$
\bar{\xi} \cdot \Re(A) \xi \geq \gamma|\xi|^{2} \quad \text { and } \quad \bar{\xi} \cdot \Im(A) \xi \leq 0 \quad \text { for all } \xi \in \mathbb{C}^{m} \text {, a.e. } x \in \bar{D},
$$

and some constant $\gamma>0$, and $n \in L^{\infty}(D)$ such that $\Re(n) \geq n_{0}>0$ and $\Im(n) \geq 0$. The far field pattern $u^{\infty}$ of the scattered field $u^{s}$ is defined via the following asymptotic expansion of the scattered field

$$
u^{s}(x)=\frac{\exp (i k r)}{r^{\frac{m-1}{2}}} u^{\infty}(\hat{x}, d)+O\left(\frac{1}{r^{\frac{m+1}{2}}}\right), r \rightarrow \infty
$$

where $\hat{x}=x /|x|$ (c.f. [5], [12]). Letting $S:=\{x:|x|=1\}$ denote the unit sphere, we assume that we know $u^{\infty}(\hat{x}, d), \hat{x} \in S$, for all incident directions $d \in S$, and define the far field operator $F: L^{2}(S) \rightarrow L^{2}(S)$ by

$$
(F g)(\hat{x}):=\int_{S} u^{\infty}(\hat{x}, d) g(d) d s(d) \text {. }
$$


We recall that

$$
F g:=u_{g}^{\infty}
$$

where $u_{g}^{\infty}$ is the far field pattern of the scattered field $u_{g}^{s}$ corresponding to (1) with $u^{i}:=v_{g}$ where $v_{g}$ is the Herglotz wave function defined by

$$
v_{g}(x):=\int_{S} e^{i k x \cdot d} g(d) d s(d)
$$

Note that the far field operator $F$ is related to the scattering operator $\mathcal{S}$ by $\mathcal{S}=I+\frac{i k}{2 \pi} F$ in $\mathbb{R}^{3}$ and by $\mathcal{S}=I+\frac{i k}{\sqrt{2 \pi k}} F$ in $\mathbb{R}^{2}$. It is well-known (see e.g. [5]) that the study of injectivity of $F$ brings to discussion the transmission eigenvalues, i.e. the values of $k \in \mathbb{C}$ such that

$$
\begin{array}{ll}
\nabla \cdot A \nabla w+k^{2} n w=0 & \text { in } D \\
\Delta v+k^{2} v=0 & \text { in } D \\
w=v & \text { on } \partial D \\
\frac{\partial w}{\partial \nu_{A}}=\frac{\partial u}{\partial \nu} & \text { on } \partial D,
\end{array}
$$

has a nontrivial solution. Under appropriate assumptions on $A$ and $n$, infinitely many transmission eigenvalues exists, in the case when $\Im(A) \neq 0$ or $\Im(n) \neq 0$ in $D$ all of them are complex (with nonzero imaginary part), and if both $A$ and $n$ are real (i.e. no absorption) there exist an infinite set of real eigenvalues (c.f. [5]). The real transmission eigenvalues can be determined from the far field operator $F[1],[7],[18]$, [20]. On the other hand the monotonicity results for real transmission eigenvalues proven in [8] open the possibility to use transmission eigenvalues to obtain information on the constitutive material properties $A$ and/or $n$ of the scattering medium [9], [13], [14], [22], [24]. Although real transmission eigenvalues are physical quantities and provide systematic quantitative information on the scattering media, their use in nondestructive testing has two major drawbacks. The first drawback is that in general only the first few transmission eigenvalues can be accurately determined from the measured data and the determination of these eigenvalue means that the frequency of the interrogating wave must be varied in a frequency range around these eigenvalues. In particular, multifrequency data must be used in an a priori determined frequency range, and since the first few transmission eigenvalues (which can be determined accurately) are determined by the material properties of the scatterer, one cannot choose the range of interrogating frequencies. The second drawback is that only real transmission eigenvalues can be determined from the measured scattering data which means that transmission eigenvalues cannot be used for the nondestructive testing of inhomogeneous absorbing media.

To deal with the above shortcomings of the use of transmission eigenvalues in non-destructive testing, in [6] the authors introduced the idea of modifying the far field operator by subtracting from the far field operator $F$ (13) for a fixed wave number $k$, the far field operator corresponding to the scattering by an impedance obstacle containing 
$D$ with constant impedance $\lambda \in \mathbb{C}$. Then the study of the injectivity of this modified far field operator yield a Steklov eigenvalue problem for $\lambda$ instead of the transmission eigenvalue problem. In [6], it was then shown following [7] that these (possibly complex) Steklov eigenvalues can be determined from the scattering data. The modification of the far field operator is not limited to the aforementioned case. In general, one could consider a one parametric family (let $\lambda$ denote this parameter) of appropriately defined scattering problems with $F_{b}^{\lambda}$ the corresponding far field operator (which can be precomputed). Then the modified far field operator $\mathcal{F}: L^{2}(S) \rightarrow L^{2}(S)$ is defined by

$$
\mathcal{F} g=F g-F_{b}^{\lambda} g, \quad g \in L^{2}(S) .
$$

This modification process can be seen as (mathematically) changing the background where the unknown inhomogeneneity is embedded, since $\mathcal{F} g$ is the far field pattern corresponding to the scattering field by the inhomogeneous media due to $v_{g}-u_{\lambda, g}^{s}$ as incident field, where $u_{\lambda, g}^{s}$ is the scattered field of the introduced scattering problem due to $v_{g}$ as incident field. Injectivity of $\mathcal{F}$ gives rise to an eigenvalue problem for $\lambda$. Note that the interrogating wave number $k$ is fixed and the eigenvalue parameter $\lambda$ is not physical, hence can be complex, which allows for applying these ideas to nondestructive testing of absorbing media. Also $F_{b}^{\lambda}$ has nothing to do with the physical scattering problem, and therefore can be pre-computed and stored. One of the goals of the current paper is to provide a general rigorous framework to determine these eigenvalues $\lambda$ from a knowledge of the modified far field operator $\mathcal{F}$. Our approach is developed within the framework of the generalized linear sampling method introduced in [1] and [2], and as oppose to [7], provides a criterion independent of the (possibly unknown) support $D$ and is mathematically justified for noisy data. We shall consider two different possibilities for the construction of $F_{b}^{\lambda}$, the one introduced in [6] in the isotropic case leading to the so-called Steklov eignevalue problem, and the another one based on the artificial scattering problem for inhomogeneous metamaterial media. The latter is related to the one discussed in [11], but here we use different sign combination for the parameters. Considering a metamaterial artificial background leads to an eigenvalue problem that has similar structure as the Steklov eignevalue problem.

The organization of the paper is as follows. In the next section we revisit the modification used in [6] and provide some new theoretical results on the related Steklov eigenvalues which can be used in obtaining information on $A$ and $n$. In Section 3 we set up the mathematical framework to apply our approach for the determination of Steklov eigenvalues from the scattering data. The latter is based on a slightly modified version of the generalized linear sampling method that we present in Appendix A. Finally, in Section 4 we introduce and study (following the lines of Section 2) the new type of transmission eigenvalue problem related to metamaterial artificial background and show how our approach can be applied to determine the related eigenvalues. We finally provide some preliminary numerical examples for this new eigenvalue problem.

We end this section with a short discussion on the scattering problem (1), recalling some results from [5] for later use. It is convenient to rewrite (1) in terms of the scattered 
field since this way we can define the scattering problem for a larger class of incident waves. In particular, for $\varphi \in L^{2}(D)^{3}$ and $\psi \in L^{2}(D)$ we define the unique function $w^{s} \in H_{l o c}^{1}\left(\mathbb{R}^{3}\right)$ satisfying

$$
\begin{gathered}
\nabla \cdot A \nabla w^{s}+k^{2} n w^{s}=\nabla \cdot(I-A) \varphi+k^{2}(1-n) \psi \quad \text { in } \mathbb{R}^{m} \\
\lim _{r \rightarrow \infty} r^{\frac{m-1}{2}}\left(\frac{\partial w^{s}}{\partial r}-i k w^{s}\right)=0 .
\end{gathered}
$$

Hence if $\psi(x)=e^{i k x \cdot d}$ and $\varphi(x)=\nabla e^{i k x \cdot d}$, then $w^{s}=u^{s}(\cdot, d)$ and the far field pattern $w^{\infty}$ of $w^{s}$ coincides with $u^{\infty}(\cdot, d)$, where $u^{s}(\cdot, d)$ and $u^{\infty}(\cdot, d)$ are the scattered field solving (1) and the corresponding, far field respectively. Furthermore, we have that $F g:=w_{g}^{\infty}$, with $w_{g}^{\infty}$ being the far field pattern of $w_{g}^{s}$ satisfying (7) with $\psi:=v_{g}, \varphi:=\nabla v_{g}$, where $v_{g}$ is the wave Herglotz function (4). Now, let $H: L^{2}(S) \rightarrow L^{2}(D) \times L^{2}(D)$ be defined by

$$
H g=\left(\left.\nabla v_{g}\right|_{D},\left.v_{g}\right|_{D}\right)
$$

and $H^{*}: L^{2}(D) \times L^{2}(D) \rightarrow L^{2}(S)$ be its $L^{2}$-adjoint which takes the form

$$
H^{*}(\varphi, \psi):=\int_{D}(-i k \hat{x} \cdot \varphi(y)+\psi(y)) e^{-i k \hat{x} \cdot y} d y .
$$

Then the far field operator $F$ assumes the following factorization

$$
F g=H^{*} T H .
$$

Here $T: L^{2}(D) \times L^{2}(D) \rightarrow L^{2}(D) \times L^{2}(D)$ is defined by

$$
T(\varphi, \psi):=-\gamma\left((A-I)\left(\varphi+\nabla w^{s}\right), k^{2}(1-n)\left(\psi+w^{s}\right)\right)
$$

where $w^{s}$ is the solution of (7) for the given $(\varphi, \psi)$, and $\gamma:=k^{2} / 4 \pi$ for $m=3$ and $\gamma:=e^{i \pi / 4} \sqrt{8 \pi k}$ for $m=2$.

\section{Steklov Eigenvalues}

In this section we give an example of the modified far field operator (6) which gives rise to Steklov eigenvalues instead of the transmission eigenvalues. This modification was first introduced in [6] for the case when $A=I$. More specifically, we consider the bounded region $D_{b} \subset \mathbb{R}^{m}$ with a piece-wise smooth boundary $\partial D_{b}$ and connected complement such that $D \subseteq D_{b}$ and introduce the scattering problem of finding $u_{b} \in H_{l o c}^{1}\left(\mathbb{R}^{m} \backslash \overline{D_{b}}\right)$ such that

$$
\begin{array}{cc}
\Delta u_{b}+k^{2} u_{b}=0 & \text { in } \mathbb{R}^{m} \backslash \overline{D_{b}} \\
u_{b}=u_{b}^{s}+u^{i} & \\
\frac{\partial u_{b}}{\partial \nu}+\lambda u_{b}=0 & \text { on } \partial D_{b} \\
\lim _{r \rightarrow \infty} r^{\frac{m-1}{2}}\left(\frac{\partial u_{b}^{s}}{\partial r}-i k u_{b}^{s}\right) & =0,
\end{array}
$$


where the incident wave $u^{i}(x):=e^{i k x \cdot d}$ is a plane wave. This problem is well-posed as long as $\lambda \in \mathbb{C}$ and $\Im(\lambda) \geq 0$. Let $u_{b}^{\infty}(\hat{x}, d)$ denote the far field pattern corresponding to $u_{b}^{s}$. The corresponding far field operator $F_{b}^{\lambda}: L^{2}(S) \rightarrow L^{2}(S)$ is given by

$$
\left(F_{b}^{\lambda} g\right)(\hat{x}):=\int_{S} u_{b}^{\infty}(\hat{x}, d) g(d) d s(d) .
$$

Note that $F_{b}^{\lambda} g=u_{b, g}^{\infty}$ is the far field pattern of the radiating solution $u_{b, g}^{s}$ solving (12) with incident wave $u^{i}:=v_{g}$, where $v_{g}$ is the wave Herglotz function (4).

Now we define the modified far field operator $\mathcal{F}: L^{2}(S) \rightarrow L^{2}(S)$ by

$$
\mathcal{F} g=F g-F_{b}^{\lambda} g .
$$

To see how the Steklov eigenvalue problem appears, we investigate the injectivity of $\mathcal{F}$. In particular, $\mathcal{F} g=0$ means that $u_{g}^{\infty}(\hat{x})=u_{b, g}^{\infty}(\hat{x}), \hat{x} \in S$ and by Rellich's lemma and unique continuation principle, $u_{g}(x)=u_{b, g}(x)$ for all $x \in \mathbb{R}^{m} \backslash D_{b}$. Hence using the boundary condition for $u_{b, g}$ on $\partial D_{b}$ and continuity of the Cauchy data for $u_{g}$ across $\partial D_{b}$, we obtain that $w:=\left.u_{g}\right|_{D_{b}}$ satisfies the boundary value problem

$$
\begin{aligned}
& \nabla \cdot A \nabla w+k^{2} n w=0 \text { in } D_{b} \\
& \frac{\partial w}{\partial \nu_{A}}+\lambda w \quad=0 \text { on } \partial D_{b} .
\end{aligned}
$$

where $A=I$ and $n=1$ in $D_{b} \backslash \bar{D}$. The solution of (15)-(16) will be identically zero unless $\lambda$ is a Steklov eigenvalue $\lambda \in \mathbb{C}$ for (15)-(16), thus implying that $u_{g}=0$ and hence $w_{g}^{s}=v_{g}$ which happens only if $g=0$ (one field is radiating the other entire solution of the Helmholtz equation). Thus if $\lambda$ is not a Steklov eigenvalues, the modified far field operator $\mathcal{F}$ is injective. Recall that in this context the Steklov eigenvalues $\lambda$ in connection with $\mathcal{F}$ appear in the same way as transmission eigenvalues $k$ in connection with $F$. Hence the question of interest in the next section is to determine these Steklov eigenvalues from a knowledge of (14), and we will do so using the framework of the generalized linear sampling method developed in [1], [2].

The above Steklov eigenvalues $\lambda$ otherwise are the eigenvalues of the Dirichlet-toNeuman operator corresponding to the equation (15). In the case when $\Im(A)=0$ and $\Im(n)=0$ the Steklov eigenvalue problem (15)-(16) is a selfadjoint eigenvalue problem for a compact operator. Obviously, if $\Im(A)<0$ or/and $\Im(n)>0$ it is not selfadjoint any longer and all the Steklov eigenvalues are complex (their existence is proven e.g. in [6] for $A=I$.) In the following we further explore the case when (15)-(16) is selfadjoint with the goal to obtain more explicit relations between Steklov eigenvalues and material properties $A, n$. To this end, we assume that $\Im(A)=0$ and $\Im(n)=0$ in $D_{b}$ and denote by

$$
\begin{aligned}
& a_{\text {min }}:=\inf _{D_{b}} \inf _{|\xi|=1} \xi \cdot A \xi>0 \text { and } \quad a_{\max }:=\sup _{D_{b}} \sup \xi \cdot A \xi \mid=1 \\
& n_{\min }:=\inf _{D_{b}}(n)>0 \quad \text { and } \quad n_{\max }:=\sup _{D_{b}}(n)<\infty .
\end{aligned}
$$


The eigenvalue problem (15)-(16) can be written as

$$
\int_{D_{b}} \nabla w \cdot A \nabla \bar{w}^{\prime} d x-k^{2} \int_{D_{b}} n w \bar{w}^{\prime} d x=-\lambda \int_{\partial D_{b}} w \bar{w}^{\prime} d s \quad \text { for all } \quad w^{\prime} \in H^{1}\left(D_{b}\right) .
$$

If $k^{2}$ is not a Robin eigenvalue, i.e. eigenvalue of

$$
\nabla \cdot A \nabla w+k^{2} n w=0 \text { in } D_{b}, \quad \frac{\partial w}{\partial \nu}+\alpha w=0 \text { on } \partial D_{b},
$$

where $0 \leq \alpha$ is fixed $((\alpha=0)$ corresponds to Neumann eigenvalue) we define the interior selfadjoint Robin-to-Dirichet operator $R: L^{2}\left(\partial D_{b}\right) \rightarrow L^{2}\left(\partial D_{b}\right)$ mapping

$$
R:\left.\theta \mapsto w_{\theta}\right|_{\partial D_{b}}
$$

where $w_{\theta} \in H^{1}(D)$ is the unique solution to

$$
\int_{D_{b}} A \nabla w_{\theta} \cdot \nabla \bar{w}^{\prime} d x+\alpha \int_{\partial D_{b}} w_{\theta} \bar{w}^{\prime}-k^{2} \int_{D_{b}} n w_{\theta} \bar{w}^{\prime} d x=\int_{\partial D_{b}} \theta \bar{w}^{\prime} d s, \quad \text { for all } w^{\prime} \in H^{1}\left(D_{b}\right) .
$$

The fact that $\left.w_{\theta}\right|_{\partial D_{b}} \in H^{1 / 2}\left(\partial D_{b}\right)$ implies that $R: L^{2}\left(\partial D_{b}\right) \rightarrow L^{2}\left(\partial D_{b}\right)$ is compact. Then $\lambda$ is a Steklov eigenvalue if and only if

$$
(-\lambda+\alpha) R \theta=\theta
$$

Note that from the analytic Fredholm theory [12], a given $k^{2}$ can not be Robin eigenvalue for all $\alpha \geq 0$. Thus, choosing $\alpha$ appropriately we have proven that for any fixed wave number $k>0$, there exists an infinite set of Steklov eigenvalues, all the eigenvalues $\lambda_{j}$ are real without finite accumulation point. In the following lemma we actually show that they accumulate only at $-\infty$. To this end, let $(\cdot, \cdot)$ denote the $L^{2}\left(D_{b}\right)$-inner product and $\langle\cdot, \cdot\rangle$ the $L^{2}\left(\partial D_{b}\right)$-inner product.

Assumption 1. The wave number $k>0$ is such that $\eta:=k^{2}$ is not a Dirichlet eigenvalue of the problem, $w \in H^{1}\left(D_{b}\right)$,

$$
\nabla \cdot A \nabla w+\eta n w=0 \quad \text { in } D_{b}, \quad w=0 \quad \text { on } \partial D_{b} .
$$

Theorem 1. For real valued $A$ and $n$ and fixed $k>0$ there exists at least one positive Steklov eigenvalue. If in addition $k>0$ satisfies Assumption 1, then there are at most finitely many positive Steklov eigenvalues.

Proof. We assume to the contrary that all eigenvalues $\lambda_{j} \leq 0$. This means that

$$
\int_{D_{b}} \nabla w \cdot A \nabla \bar{w} d x-k^{2} \int_{D_{b}} n|w|^{2} d s \geq 0
$$

for all $w \in H^{1}\left(D_{b}\right)$ since the Steklov eigenfunctions form a Riesz basis for $H^{1}\left(D_{b}\right)$. Now taking $w=1$ yields a contradiction which proves the first statement. 
Next we assume by contradiction that there exists a sequence of positive Steklov eigenvalues $\lambda_{j}>0, j \in \mathbb{N}$ converging to $+\infty$ with eigenfunction $w_{j}$ normalized such that

$$
\left\|w_{j}\right\|_{H^{1}\left(D_{b}\right)}+\left\|w_{j}\right\|_{L^{2}\left(\partial D_{b}\right)}=1
$$

Then from

$$
\left(A \nabla w_{j}, \nabla w_{j}\right)-k^{2}\left(n w_{j}, w_{j}\right)=-\lambda_{j}\left\langle w_{j}, w_{j}\right\rangle
$$

since the left hand side is bounded we obtain that $w_{j} \rightarrow 0$ in $L^{2}\left(\partial D_{b}\right)$. Next, up to a subsequence $w_{j}$ converges weakly in $H^{1}\left(D_{b}\right)$ to some $w \in H^{1}\left(D_{b}\right)$ and this weak limit satisfies $\nabla \cdot A \nabla w+k^{2} n w=0$ in $D_{b}$ and and from the above $w=0$ on $\partial D_{b}$. Therefore, using Assumption 1, $w=0$ in $D_{b}$. Hence, up to a subsequence, $w_{j} \rightarrow 0$ in $L^{2}\left(D_{b}\right)$ (strongly). From (22)

$$
\left(A \nabla w_{j}, \nabla w_{j}\right)-k^{2}\left(n w_{j}, w_{j}\right)<0, \quad \text { for all } j \in \mathbb{N}
$$

and since the left hand side is a bounded real sequence, we can conclude that up to a subsequence

$$
\left(A \nabla w_{j}, \nabla w_{j}\right) \rightarrow 0, \quad \text { as } j \rightarrow \infty
$$

which implies that $\left\|\nabla w_{j}\right\|_{L^{2}\left(D_{b}\right)} \rightarrow 0$ in addition to $\left\|w_{j}\right\|_{L^{2}\left(\partial D_{b}\right)} \rightarrow 0$. This contradicts $(21)$.

For the existence of Steklov eigenvalues for complex valued $C^{\infty}$ coefficient $n(x)$ and $A=I$ see [6]. The approach there can be easily generalized to the case of $A \neq I$ with $C^{\infty}$ coefficients (see also [25]).

We let $\tau_{0}:=\tau_{0}\left(D_{b}, \alpha\right)$, for $0<\alpha<\infty$ be the first Robin eigenvalue of

$$
\begin{aligned}
& \Delta u+\tau u=0 \text { in } D_{b}, \quad \frac{\partial u}{\partial \nu}+\alpha u=0 \text { on } \partial D_{b}, \\
& \tau_{0}=\inf _{u \in H^{1}\left(D_{b}\right), u \neq 0} \frac{(\nabla u, \nabla u)+\alpha\langle u, u\rangle}{(w, w)} .
\end{aligned}
$$

(Note that the ball $B$ with the same volume as $D_{b}$ and a particular constant $\alpha$ minimizes $\tau_{0}\left(D_{b}, \alpha\right)$, see [16].) Next we will try to choose a positive constant $\Lambda>0$, such that

$$
\int_{D_{b}} \nabla w \cdot A \nabla \bar{w} d x-k^{2} \int_{D_{b}} n|w|^{2} d x+\Lambda \int_{\partial D_{b}}|w|^{2} d s \geq c\|w\|_{H^{1}\left(D_{b}\right)}^{2}, \quad c>0
$$

Indeed, using (24)

$$
\begin{aligned}
\int_{D_{b}} \nabla w & \cdot A \nabla \bar{w} d x-k^{2} \int_{D_{b}} n|w|^{2} d x+\Lambda \int_{\partial D_{b}}|w|^{2} d s \\
\geq & \left(a_{\min }-\frac{k^{2} n_{\max }}{\tau_{0}}\right) \int_{D_{b}}|\nabla w|^{2} d x+\left(\Lambda-\frac{k^{2} n_{\max }}{\tau_{0}} \alpha\right) \int_{\partial D_{b}}|w|^{2} d s
\end{aligned}
$$


we can find such a $\Lambda$ assuming that $\tau_{0} a_{\min }-k^{2} n_{\max }>0$. Hence in this case our eigenvalue problem, which can be written as

$$
\int_{D_{b}} \nabla w \cdot A \nabla \bar{w}^{\prime} d x-k^{2} \int_{D_{b}} n w \bar{w}^{\prime} d x+\Lambda \int_{\partial D_{b}} w \bar{w}^{\prime} d s=-(\lambda-\Lambda) \int_{\partial D_{b}} w \bar{w}^{\prime} d s .(26)
$$

becomes a generalized eigenvalue problem for a positive selfadjoint compact operator and hence the eigenvalues $\Lambda-\lambda>0$ satisfy the Courant-Fischer inf-sup principle (see e.g. Chapter 4 in [5]). In particular, if $\tau_{0} a_{\text {min }}-k^{2} n_{\max }>0$ the largest positive Steklov eigenvalue $\lambda_{1}=\lambda_{1}(A, n, k)$ satisfies

$$
\lambda_{1}=\sup _{w \in H^{1}\left(D_{b}\right), w \neq 0} \frac{k^{2} \int_{D_{b}} n|w|^{2} d x-\int_{D_{b}} \nabla w \cdot A \nabla w d x}{\int_{\partial D_{b}}|w|^{2} d s},
$$

whence it depends monotonically increasing with respect $n$ and monotonically decreasing with respect to $A$. We obtain here a conditional monotonicity property for the largest positive Steklov eigenvalue. In the following theorem we give the optimal condition on $A, n$ and $k$ which ensure the coercivity property (25), whence the sup-condition (27).

Theorem 2. Assume that $k^{2}<\eta_{0}\left(A, n, D_{b}\right)$, where $\eta_{0}\left(A, n, D_{b}\right)$ is the first Dirichlet eigenvalue of (20). Then there is a $\Lambda>0$ such that (25) holds. In particular, the largest positive Steklov eigenvalue satisfies (27).

Proof. Fix $k^{2}<\eta_{0}\left(A, n, D_{b}\right)$ and assume to the contrary that there exists a sequence of positive constants $\Lambda_{j}=j, j \in \mathbb{N}$, and a sequence of functions $w_{j} \in H^{1}\left(D_{b}\right)$ normalized as $\left\|w_{j}\right\|_{H^{1}\left(D_{b}\right)}=1$ such that

$$
\int_{D_{b}} \nabla w_{j} \cdot A \nabla \bar{w}_{j} d x-k^{2} \int_{D_{b}} n\left|w_{j}\right|^{2} d x+j \int_{\partial D_{b}}\left|w_{j}\right|^{2} d s \leq 0 .
$$

From

$$
\int_{D_{b}} \nabla w_{j} \cdot A \nabla \bar{w}_{j} d x+j \int_{\partial D_{b}}\left|w_{j}\right|^{2} d s \leq k^{2} \int_{D_{b}} n\left|w_{j}\right|^{2} d x
$$

we see that $j \int_{\partial D_{b}}\left|w_{j}\right|^{2} d s$ is bounded which implies that $w_{j} \rightarrow 0$ strongly in $L^{2}\left(\partial D_{b}\right)$ as $j \rightarrow+\infty$. On the other hand the boundedness implies that $w_{j} \rightarrow w$ weakly in $H^{1}\left(D_{b}\right)$ and from the above $w=0$ on $\partial D_{b}$, whence $w \in H_{0}^{1}\left(D_{b}\right)$. Next, we have that up to a subsequence $w_{j} \rightarrow w$ strongly in $L^{2}\left(D_{b}\right)$. Since the norm of the weak limit is smaller that the lim-inf of the norm

$$
(A \nabla w, \nabla w) \leq \liminf _{j \rightarrow \infty} \int_{D_{b}} \nabla w_{j} \cdot A \nabla \bar{w}_{j} d x \leq \lim _{j \rightarrow \infty} k^{2} \int_{D_{b}} n\left|w_{j}\right|^{2} d x=k^{2}(n w, w)
$$

which contradicts the fact that

$$
k^{2}<\inf _{w \in H_{0}^{1}\left(D_{b}\right), w \neq 0} \frac{(A \nabla w, w)}{(n w, w)}=\eta_{0}\left(A, n, D_{b}\right) .
$$

This ends the proof. 
In [6] for the case of $A=I$ it is shown by an example that Steklov eigenvalues $\lambda:=\lambda(k)$ as a function of $k$ can blow up as $k$ approaches a Dirichlet eigenvalue defined in Assumption 1. We prove this in general for the largest positive Steklov eigenvalues and as $k$ approaches the first Dirichlet eigenvalue $\eta_{0}\left(A, n, D_{b}\right)$.

Theorem 3. Assume that $k^{2}<\eta_{0}\left(A, n, D_{b}\right)$, where $\eta_{0}\left(A, n, D_{b}\right)$ is the first Dirichlet eigenvalue of (20). Then the largest positive Steklov eigenvalue $\lambda_{1}=\lambda_{1}(k)$ as a function of $k$ approaches $+\infty$ as $k^{2} \rightarrow \eta_{0}\left(A, n, D_{b}\right)$.

Proof. Consider the first eigenvalue and eigenvector $\left(\eta_{\delta}, w_{\delta}\right),\left\|w_{\delta}\right\|_{H^{1}\left(D_{b}\right)}=1$, of the following Robin problem

$$
\nabla \cdot A \nabla w_{\delta}+\eta_{\delta} n w_{\delta}=0 \text { in } D_{b}, \quad \frac{\partial w_{\delta}}{\partial \nu_{A}}+\frac{1}{\delta} w_{\delta}=0 \text { on } \partial D_{b} .
$$

for $\delta>0$. If $\eta_{0}:=\eta_{0}\left(A, n, D_{b}\right)$ and $w_{0}$ denote the first Dirichlet eigenvalue and eigenvector of $(20)$, we notice that

$$
\begin{aligned}
\eta_{\delta}=\frac{\left(A \nabla w_{\delta}, w_{\delta}\right)+\frac{1}{\delta}\left\langle w_{\delta}, w_{\delta}\right\rangle}{\left(n w_{\delta}, w_{\delta}\right)} & =\inf _{w \in H^{1}\left(D_{b}\right), w \neq 0} \frac{(A \nabla w, w)+\frac{1}{\delta}\langle w, w\rangle}{(n w, w)} \\
& <\inf _{w \in H_{0}^{1}\left(D_{b}\right), w \neq 0} \frac{(A \nabla w, w)}{(n w, w)}=\eta_{0}
\end{aligned}
$$

i.e. $\eta_{\delta}<\eta_{0}$. Using the inf criterion, one also easily observe that $\delta \mapsto \eta_{\delta}$ is decreasing, whence $\lim _{\delta \rightarrow 0} \eta_{\delta}$ exits. On the other hand, (29) can be written as

$$
\int_{D_{b}} A \nabla w_{\delta} \cdot \nabla \bar{w}^{\prime} d x+\frac{1}{\delta} \int_{\partial D_{b}} w_{\delta} \bar{w}^{\prime} d s=\eta_{\delta} \int_{D_{b}} n w_{\delta} \bar{w}^{\prime} d x,
$$

and by taking $w^{\prime}=w_{\delta}$ we see that $w_{\delta} \rightarrow 0$ strongly in $L^{2}\left(\partial D_{b}\right)$ as $\delta \rightarrow 0$. The $H^{1}\left(D_{b}\right)$ weak limit of $w_{\delta}$, denoted $w$, satisfies $\nabla \cdot A \nabla w+\left(\lim _{\delta \rightarrow 0} \eta_{\delta}\right) n w=0$ in $D_{\delta}$ and $w=0$ on $\partial D_{b}$, which means $\lim _{\delta \rightarrow 0} \eta_{\delta}=\eta_{0}$ (since $\eta_{\delta}<\eta_{0}$ and $\eta_{0}$ is the first Dirichlet eigenvalue) and $w=w_{0}$ the corresponding eigenfuction. From the compact embedding of $H^{1}\left(D_{b}\right)$ into $L^{2}\left(D_{b}\right)$ we have that (up to a subsequence) $w_{\delta} \rightarrow w_{0}$ strongly in $L^{2}\left(D_{b}\right)$. Now we consider the sequence $k_{\delta}^{2}:=\eta_{\delta}+\left\|w_{\delta}\right\|_{L^{2}\left(\partial D_{b}\right)}^{2} \rightarrow \eta_{0}$ as $\delta \rightarrow 0$. Then from (27)

$$
\begin{aligned}
\lambda_{1}\left(k_{\delta}\right) \geq & \frac{k_{\delta}^{2} \int_{D_{b}} n\left|w_{\delta}\right|^{2} d x-\int_{D_{b}} \nabla w_{\delta} \cdot A \nabla w_{\delta} d x}{\int_{\partial D_{b}}\left|w_{\delta}\right|^{2} d s} \\
= & \frac{\left(k_{\delta}^{2}-\eta_{\delta}\right) \int_{D_{b}} n\left|w_{\delta}\right|^{2} d x}{\int_{\partial D_{b}}\left|w_{\delta}\right|^{2} d s}+\frac{1}{\delta}=\int_{D_{b}} n\left|w_{\delta}\right|^{2} d x+\frac{1}{\delta} .
\end{aligned}
$$

Thus we have that

$$
\lim _{\delta \rightarrow 0} \lambda_{1}\left(k_{\delta}\right) \geq \int_{D_{b}} n\left|w_{0}\right|^{2} d x+\lim _{\delta \rightarrow 0} \frac{1}{\delta}=+\infty
$$


which ends the proof.

In the next section we show how to determine (possibly complex) Steklov eigenvalues from a knowledge of the modified far field operator. To this end, we need to recall some results from [10], [17], [19] on an appropriate factorization of $F_{b}^{\lambda}$. In particular, it is shown that $F_{b}^{\lambda}: L^{2}(S) \rightarrow L^{2}(S)$ can be factorized as

$$
F_{b}^{\lambda}=H_{b}^{*} T_{b} H_{b}
$$

where $H_{b}: L^{2}(S) \rightarrow H^{-1 / 2}\left(\partial D_{b}\right)$ is given by

$$
H_{b} g=\frac{\partial v_{g}}{\partial \nu}+\left.\lambda v_{g}\right|_{\partial D_{b}}
$$

and its conjugate dual operator $H_{b}^{*}: H^{1 / 2}\left(\partial D_{b}\right) \rightarrow L^{2}(S)$ takes the form

$$
H_{b}^{*} \varphi:=\int_{\partial D_{b}}\left(\frac{\partial e^{-i k \hat{x} \cdot y}}{\partial \nu}+\bar{\lambda} e^{-i k \hat{x} \cdot y}\right) \varphi(y) d s_{y} .
$$

The middle operator $T_{b}: H^{-1 / 2}\left(\partial D_{b}\right) \rightarrow H^{1 / 2}\left(\partial D_{b}\right)$ is the inverse of the operator $T_{b}^{-1}: H^{1 / 2}\left(\partial D_{b}\right) \rightarrow H^{-1 / 2}\left(\partial D_{b}\right)$ defined by

$$
\begin{aligned}
\left(T_{b}^{-1} \phi\right)(x) & =i \Im(\lambda) \phi(x)+\frac{\partial}{\partial \nu_{x}} \int_{\partial D_{b}} \phi(y) \frac{\partial \Phi(x, y)}{\partial \nu_{y}} d s_{y}+\lambda \bar{\lambda} \int_{\partial D_{b}} \phi(y) \Phi(x, y) d s_{y} \\
& +\int_{\partial D_{b}} \phi(y)\left[\lambda \frac{\partial \Phi(x, y)}{\partial \nu_{y}}-\bar{\lambda} \frac{\partial \Phi(x, y)}{\partial \nu_{x}}\right] d s_{y}
\end{aligned}
$$

where the radiating fundamental solution $\Phi(\cdot, \cdot)$ of the Helmholtz equation in $\mathbb{R}^{m}$ is

$$
\Phi(x, z):=\left\{\begin{array}{cc}
\frac{e^{i k|x-z|}}{4 \pi|x-z|} & \text { in } \mathbb{R}^{3} \\
\frac{i}{4} H_{0}^{(1)}(k|x-z|) & \text { in } \mathbb{R}^{2}
\end{array}\right.
$$

with $H_{0}^{(1)}$ denoting the Hankel function of the first kind of order zero. Furthermore, we can factorize

$$
\mathcal{F}=\mathcal{G H}
$$

Here $\mathcal{H}: L^{2}(S) \rightarrow H^{1 / 2}\left(\partial D_{b}\right) \times H^{-1 / 2}\left(\partial D_{b}\right)$ is defined by

$$
\mathcal{H}(g):=\left(u_{b, g}, \frac{\partial u_{b, g}}{\partial \nu}\right)_{\partial D_{b}}=\left(u_{b, g},-\lambda u_{b, g}\right)_{\partial D_{b}}
$$

where $u_{b, g}$ solves (12) with incident wave $u^{i}:=v_{g}$ the Herglotz wave function defined by (4). The operator $\mathcal{G}: \overline{\mathcal{R}(\mathcal{H})} \subset H^{1 / 2}\left(\partial D_{b}\right) \times H^{-1 / 2}\left(\partial D_{b}\right) \rightarrow L^{2}(S)$ is such that

$$
\mathcal{G}(\varphi, \psi)=w^{\infty}
$$


where $w^{\infty}$ is the far field of $w^{s}$ that solves

$$
\begin{array}{ll}
\Delta w^{s}+k^{2} w^{s}=0 & \text { in } \mathbb{R}^{m} \backslash \overline{D_{b}} \\
\nabla \cdot A \nabla w+k^{2} n w=0 & \text { in } D_{b} \\
w-w^{s}=\varphi & \text { on } \partial D_{b} \\
\frac{\partial w}{\partial \nu_{A}}-\frac{\partial w^{s}}{\partial \nu}=\psi & \text { on } \partial D_{b} \\
\lim _{r \rightarrow \infty} r^{\frac{m-1}{2}}\left(\frac{\partial w^{s}}{\partial r}-i k w^{s}\right)=0 . &
\end{array}
$$

and $\overline{\mathcal{R}(\mathcal{H})}$ is the closure of the range of $\mathcal{H}$ in $H^{1 / 2}\left(\partial D_{b}\right) \times H^{-1 / 2}\left(\partial D_{b}\right)$.

\section{Determination of Steklov Eigenvalues from Far Field Data}

In this section we discuss the determination of the Steklov eigenvalues from a knowledge of the (computable) family of operators $F_{b}^{\lambda}$ and the (measured) data operator $F$. The method relies on the abstract framework of the generalized linear sampling method given in Theorem 8 in Appendix A applied to the modified far field operator $\mathcal{F}=F-F_{b}^{\lambda}$. To this end, let $\mathcal{H}$ and $\mathcal{G}$ be defined by (35) and (36), respectively and recall that $\mathcal{F}=\mathcal{G H}$. Referring to Theorem 8 in Appendix A, here we have $X=X^{*}:=L^{2}(S)$ and $Y:=H^{1 / 2}\left(\partial D_{b}\right) \times H^{-1 / 2}\left(\partial D_{b}\right)$. There are two main points we must specify: the choice of the test function $\phi \in L^{2}(S)$ and the choice of the operator $B$ (given in terms of $F$ and $F_{b}^{\lambda}$ ) that satisfies Assumption 3 in Appendix A. We have two possibilities discussed in the lemma below.

Lemma 1. Recall $F_{b}^{\lambda}$ given by (13) and $F$ given by (2). Then either one of the following choices for $B$ satisfies Assumption 3 with $H:=\mathcal{H}$ given by (35):

(i) $B(g)=\left|\left(F_{b}^{\lambda} g, g\right)\right|$ if $D \subseteq D_{b}$ and $\lambda$ is not an eigenvalue associated with the problem: $w \in H^{1}\left(D_{b}\right)$,

$$
\Delta w+k^{2} w=0 \quad \text { in } D_{b} \quad \text { and } \quad \frac{\partial w}{\partial \nu}+\lambda w=0 \text { on } \partial D_{b} .
$$

(ii) $B(g)=|(F g, g)|$ if $D=D_{b}$ and the operator $T$ given by (11) is coercive on $\mathcal{R}(H)$ where $H$ is defined by (8).

Proof. Let us first consider the case $B(g)=\left|\left(F_{b}^{\lambda} g, g\right)\right|$. Consider a sequence $\left\{g_{n}\right\}$ such that the sequence $B\left(g_{n}\right)$ is bounded. We recall that the operator $T_{b}$ given by (32) is coercive if $\lambda$ is not an eigenvalue of (37) (see e.g. Theorem 2.6 in [17]). From factorization (31) and the coercivity of $T_{b}$ we have that $B\left(g_{n}\right)=\left|\left(F_{b}^{\lambda} g_{n}, g_{n}\right)\right|=\left|\left(T_{b} H_{b} g_{n}, H_{b} g_{n}\right)\right| \geq$ $\mu\left\|H_{b} g_{n}\right\|_{H^{-1 / 2}\left(\partial D_{b}\right)}$. Since (12) is well-posed, we have that the sequence $u_{b, g_{n}}$ is bounded in $H^{1}\left(K \backslash D_{b}\right)$ for any compact $K$ containing $D_{b}$. Hence the sequence $\mathcal{H} g_{n}$ is also bounded in $H^{1 / 2}\left(\partial D_{b}\right) \times H^{-1 / 2}\left(\partial D_{b}\right)$.

We now consider the converse implication. We first observe that since $T_{b}$ is a bounded operator, we have that $B(g)=\left|\left(F_{b}^{\lambda} g, g\right)\right|=\left|\left(T_{b} H_{b} g, H_{b} g\right)\right| \leq\left\|T_{b}\right\|\left\|H_{b} g\right\|_{H^{-1 / 2}\left(\partial D_{b}\right)}$. 
Therefore, if a sequence $H_{b} g_{n}$ is bounded then the sequence $B\left(g_{n}\right)$ is also bounded. For $g:=g_{n}$, using the Green formula and the fact that $v_{g}$ is a solution of Helmholtz equation we have that

$$
u_{b, g}^{s}(x)=\int_{\partial D_{b}}\left(u_{b, g}(y) \frac{\partial \Phi(x, y)}{\partial \nu}+\lambda u_{b, g}(y) \Phi(x, y)\right) d s_{y} .
$$

Therefore if $\mathcal{H} g_{n}$ is a bounded sequence then the scattered field $u_{b, g_{n}}^{s}$ is bounded in $H^{1}\left(K \backslash \bar{D}_{b}\right)$ for any compact set $K$ containing $D_{b}$. Therefore the sequence $H_{b} g_{n}=$ $\frac{\partial v_{g_{n}}}{\partial \nu}+\left.\lambda v_{g_{n}}\right|_{\partial D_{b}}$ is bounded in $H^{-1 / 2}\left(\partial D_{b}\right)$ and so is the sequence $B\left(g_{n}\right)$ (using the arguments above).

Now we consider the case $B(g)=|(F g, g)|$ and assume that the sequence $B\left(g_{n}\right)$ is bounded. Factorization (10) and the coercivity of $T$ give $B\left(g_{n}\right)=\left|\left(F g_{n}, g_{n}\right)\right|=$ $\left|\left(T H g_{n}, H g_{n}\right)\right| \geq \mu\left\|H g_{n}\right\|_{L^{2}(D) \times L^{2}(D)}$. The fact that (12) is well-posed implies that $u_{b, g_{n}}$ is bounded in $H_{l o c}^{1}\left(\mathbb{R}^{m} \backslash D\right)$ norm and hence $\mathcal{H} g_{n}$ is also bounded in $H^{1 / 2}(\partial D) \times$ $H^{-1 / 2}(\partial D)$. On the other hand, since $T$ is a bounded operator, we have that $B\left(g_{n}\right)=\left|\left(F g_{n}, g_{n}\right)\right|=\left|\left(T H g_{n}, H g_{n}\right)\right| \leq\|T\|\left\|H g_{n}\right\|_{L^{2}(D) \times L^{2}(D)}$, hence if $H g_{n}$ is a bounded sequence, then the sequence $B\left(g_{n}\right)$ is also bounded. Similar arguments as in the second half of the proof of the first part show that if $\mathcal{H} g_{n}$ is a bounded sequence then the sequence $H g_{n}$ is bounded and therefore the sequence $B\left(g_{n}\right)$ is bounded. The proof is completed.

Remark 1. We observe that the operator $T$ given by (11) is coercive if $k$ is not an transmission eigenvalue for (5) and a fixed sign assumption is made on the coefficients $A-I$ and $n-1$ in a neighborhood of the boundary of $\partial D$ (see e.g Theorem 2.42 in [5]). We also indicate that for more complex configurations, e.g. $D_{b} \nsubseteq D$, one could possibly consider $B(g)=\left|\left(F_{b}^{\lambda} g, g\right)\right|+|(F g, g)|$.

Lemma 1 provides us with practical choices for $B(g)$ in order to apply the abstract framework in Appendix A. For sake of presentation let us restrict ourselves to the case of $B(g)=\left|\left(F_{b}^{\lambda} g, g\right)\right|$. The choice of $B(g)=|(F g, g)|$ can be handled in a similar way. The goal is to apply Theorem 8 in Appendix A to the cost functional

$$
J_{\alpha}\left(\Phi_{z}^{\infty}, g\right)=\alpha\left(F_{b}^{\lambda} g, g\right)+\left\|\mathcal{F} g-\Phi_{z}^{\infty}\right\|^{2},
$$

where $\Phi_{z}^{\infty}$ is the far field of the fundamental solution of Helmholtz equation $\Phi(\cdot, z)$ defined by (33). The choice of $\varphi:=\Phi_{z}^{\infty}$ is motivated by the following two lemmas.

Lemma 2. Assume that $\lambda$ is not a Steklov eigenvalue of (15)-(16). Then $\Phi_{z}^{\infty} \in \mathcal{R}(\mathcal{G})$ for $z \in D_{b}$.

Proof. Fix a $z \in D_{b}$ and let $w_{z} \in H^{1}\left(D_{b}\right)$ be the unique solution of

$$
\begin{aligned}
& \nabla \cdot A \nabla w_{z}+k^{2} n w_{z}=0 \quad \text { in } D_{b} \\
& \frac{\partial w_{z}}{\partial \nu_{A}}+\lambda w_{z} \quad=\frac{\partial \Phi(\cdot, z)}{\partial \nu_{A}}+\lambda \Phi(\cdot, z) \quad \text { on } \partial D_{b} .
\end{aligned}
$$


An application of the Green representation formula implies the following splitting of $w_{z}$

$$
w_{z}=w_{z}^{s}+v_{z}
$$

where

$$
v_{z}(x):=\int_{\partial D_{b}}\left(\frac{\partial w_{z}(y)}{\partial \nu_{A}} \Phi(x, y)-w_{z}(y) \frac{\partial \Phi(x, y)}{\partial \nu}\right) d s_{y}
$$

solves the Helmholtz equation $\Delta v_{z}+k^{2} v_{z}=0$ in $D_{b}$. Now let $u_{b, z}$ be the solution of (12) with incident wave $u^{i}:=v_{z}$. Then by construction $\mathcal{G}\left(\varphi_{z}, \psi_{z}\right)=\Phi_{z}^{\infty}$ where $\varphi_{z}:=\left.u_{b, z}\right|_{\partial D_{b}}$ and $\psi_{z}=\left.\lambda u_{b, z}\right|_{\partial D_{b}}$.

Lemma 3. Assume that $\lambda$ is a Steklov eigenvalue of (15)-(16) and $\lambda$ is not an eigenvalue of (37). Then the set of points z such that $\Phi_{z}^{\infty} \in \mathcal{R}(\mathcal{G})$ is nowhere dense in $D_{b}$.

Proof. Assume to the contrary that $\Phi_{z}^{\infty} \in \mathcal{R}(\mathcal{G})$ for $z$ in a dense subset of a ball $B$ included in $D_{b}$. Thus there exists $\left(\varphi_{z}, \psi_{z}\right) \in \overline{\mathcal{R}(\mathcal{H})}$ such that $\mathcal{G}\left(\varphi_{z}, \psi_{z}\right)=\Phi_{z}^{\infty}$.

Following similar arguments as in the proof of Lemma 2.1 of [5], one obtains that if $\lambda$ is not an eigenvalue of $(37)$ then a pair $(\varphi, \psi) \in \overline{\mathcal{R}(\mathcal{H})}$ is such that $\varphi:=\left.u_{b}\right|_{\partial D_{b}}$ and $\psi=-\left.\lambda u_{b}\right|_{\partial D_{b}}$ where $u_{b}$ solves (12) with incident wave $u^{i}:=v$ for $v \in H_{\text {inc }}$ where

$$
H_{\text {inc }}:=\left\{v \in H^{1}\left(D_{b}\right): \Delta v+k^{2} v=0\right\} .
$$

We therefore infer that $\varphi_{z}:=\left.u_{b, z}\right|_{\partial D_{b}}$ and $\psi_{z}=-\left.\lambda u_{b, z}\right|_{\partial D_{b}}$ where $u_{b, z}$ is the solution of (12) with incident wave $u^{i}:=v_{z}$ for some $v_{z} \in H_{\text {inc }}$. From the definition of $\mathcal{G}(36)$ and using Rellich lemma we conclude that the corresponding $w_{z}$ satisfies

$$
\begin{array}{ll}
\nabla \cdot A \nabla w_{z}+k^{2} n w_{z}=0 & \text { in } D_{b} \\
\frac{\partial w_{z}}{\partial \nu_{A}}+\lambda w_{z}=\frac{\partial \Phi(\cdot, z)}{\partial \nu}+\lambda \Phi(\cdot, z) & \text { on } \partial D_{b} .
\end{array}
$$

From the Fredholm alternative, the above problem is solvable if and only if

$$
\int_{\partial D_{b}}\left(\frac{\partial \Phi(\cdot, z)}{\partial \nu}+\lambda \Phi(\cdot, z)\right) \bar{w}_{\lambda} d s=0
$$

where $w_{\lambda}$ is in the kernel of the adjoint problem, i.e. satisfied

$$
\begin{aligned}
\nabla \cdot \bar{A} \nabla w_{\lambda}+k^{2} \bar{n} w_{\lambda} & =0 & & \text { in } D_{b} \\
\frac{\partial w_{\lambda}}{\partial \nu_{\bar{A}}}+\bar{\lambda} w_{\lambda} & =0 & & \text { on } \partial D_{b} .
\end{aligned}
$$

Using the boundary conditions for $w_{\lambda}$ on $\partial D_{b}$, the equation (41) then gives

$$
v_{\lambda}(z):=\int_{\partial D_{b}}\left(\frac{\partial \Phi(\cdot, z)}{\partial \nu} \bar{w}_{\lambda}-\Phi(\cdot, z) \frac{\partial \bar{w}_{\lambda}}{\partial \nu_{A}}\right) d s=0
$$

for $z$ in $B$. Since $v_{\lambda}$ satisfies the Helmholtz equation in $D_{b}$, then $v_{\lambda}=0$ in $D_{b}$. Let us define

$$
w_{\lambda}^{s}:=\bar{w}_{\lambda}-v_{\lambda} \text { in } D_{b}
$$


and

$$
v_{\lambda}^{s}(x):=\int_{\partial D_{b}}\left(\frac{\partial \Phi(\cdot, x)}{\partial \nu} \bar{w}_{\lambda}-\Phi(\cdot, x) \frac{\partial \bar{w}_{\lambda}}{\partial \nu_{A}}\right) d s \quad x \in \mathbb{R}^{m} \backslash \overline{D_{b}} .
$$

Then $w_{\lambda}^{s}$ is a solution of (1) with $D=D_{b}$ and $u^{i}=0$. Therefore $w_{\lambda}^{s}=0$ and then $w_{\lambda}=0$ in $D_{b}$. This gives a contradiction.

We are now ready to apply Theorem 8 in Appendix A to the operator $\mathcal{F}$ based on the fundamental results of Lemma 2 and Lemma 3. To this end we need that $\mathcal{F}$ has dense range which is obviously the case if $\lambda$ is not a Steklov eigenvalue. Hence we add the following assumption.

Assumption 2. Assume that $\mathcal{F}$ has still dense range at $\lambda$ a Steklov eigenvalue of (15)(16).

This assumption means that if $\lambda$ a Steklov eigenvalue then the corresponding Steklov eigenvector should not be of the form $v_{g}+u_{b, g}^{s}$, with $v_{g}$ being a Herglotz wave function. Since the latter is a special representation that would only hold in particular configurations of the domain $D_{b}$ (for instance spherically symmetric configurations), Assumption 2 is then expected to be generically true.

Combining Theorem 8, Lemma 2 and Lemma 3 we obtain the main result of this section.

Theorem 4. Assume that the modified far field operator $\mathcal{F}: L^{2}(S) \rightarrow L^{2}(S)$ satisfies Assumption 2, $D \subset D_{b}$ and $\lambda$ is not an eigenvalue of the problem (37). Consider the functional

$$
J_{\alpha}\left(\Phi_{z}^{\infty}, g\right):=\alpha\left|\left(F_{b}^{\lambda} g, g\right)\right|+\left\|\mathcal{F} g-\Phi_{z}^{\infty}\right\|^{2} \quad \text { and } \quad j_{\alpha}\left(\Phi_{z}^{\infty}\right):=\inf _{g} J_{\alpha}\left(\Phi_{z}^{\infty}, g .\right)
$$

Let $g_{\alpha}^{z}$ be a minimizing sequence defined by

$$
J_{\alpha}\left(\Phi_{z}^{\infty}, g_{\alpha}^{z}\right) \leq j_{\alpha}\left(\Phi_{z}^{\infty}\right)+C \alpha
$$

where $C>0$ is a constant independent of $\alpha>0$. Then a complex number $\lambda \in \mathbb{C}$ is a Steklov eigenvalue of (15)-(16) if and only if the set of points z such that $\left|\left(F_{b}^{\lambda} g_{\alpha}^{z}, g_{\alpha}^{z}\right)\right|$ is bounded as $\alpha \rightarrow 0$ is nowhere dense in $D_{b}$.

Remark 2. The use of the indicator function $\left|\left(F_{b}^{\lambda} g_{\alpha}^{z}, g_{\alpha}^{z}\right)\right|$ has the advantage of treating the case when $D \subset D_{b}$ but on the other had requires that the problem (37) is uniquely solvable. The latter can be avoided in the case of $D=D_{b}$ by choosing $B(g)=|(F g, g)|$, whence using the indicator function $\left|\left(F g_{\alpha}^{z}, g_{\alpha}^{z}\right)\right|$, but in this case $k$, which is fixed, cannot be an interior transmission eigenvalue for (5).

We end this section by commenting that a similar rigorous characterization of Steklov eigenvalues as in Theorem 4 can also be obtained for the noisy data. The modification of Theorem 8 in Appendix A for the case of noisy data is considered in details in [2] (see also [1] and [5]). All the results presented here can apply to the case of noisy operators $F^{\delta}, F_{b}^{\lambda, \delta}$ and $\mathcal{F}^{\delta}$, where $\delta$ denotes the noise level in the measurements 
of the far field data. In this case, one simply has to consider instead the modified (regularized) cost function

$$
J_{\alpha}^{\delta}\left(\Phi_{z}^{\infty}, g\right)=\alpha\left|\left(F_{b}^{\lambda, \delta} g, g\right)\right|+\alpha \delta\|g\|^{2}+\left\|\mathcal{F}^{\delta} g-\Phi_{z}^{\infty}\right\|^{2} .
$$

Then the criteria is in terms of the modified indicator function

$$
\lim _{\alpha \rightarrow 0} \lim _{\delta \rightarrow 0}\left[\left|\left(F_{b}^{\lambda, \delta} g_{\alpha \delta}^{z}, g_{\alpha \delta}^{z}\right)\right|+\delta\left\|g_{\alpha \delta}^{z}\right\|^{2}\right] .
$$

For a priori choice of $\alpha$ in terms to $\delta$ under some restrictive assumptions we refer the reader [2], while noting that in general such a choice remains still an open problem.

Remark 3. If limited aperture data is available, i.e. $u^{\infty}(\hat{x}, d)$ is known for $\hat{x} \in S_{r}$ and $d \in S_{t}$ where (the transmitters location) $S_{t}$ and (the receivers location) $S_{r}$ are open subsets of the unit sphere $S$, the above discussion is valid if $F$ is replaced by

$$
(F g)(\hat{x}):=\int_{S_{t}} u_{\infty}(\hat{x}, d) g(d) d s(d), \quad \hat{x} \in S_{r}
$$

(we refer the reader to [3] for the theoretical foundations of GLSM with limited aperture data). In this case the indicator function $\left|\left(F_{b}^{\lambda} g, g\right)\right|$ may have advantage in practice because, thanks to the fact that $F_{b}^{\lambda}$ is computed, a symmetric factorization for it is always available. However numerical experiments are needed to study the sensitivity of the determination of the eigenvalues $\lambda$ in terms of the aperture of the data.

\section{Artificial Metamaterial Background}

Next we turn our attention to a alternative example of modifying the far field operator which leads to a new eigenvalue problem whose eigenvalues can also be determined using the analytical framework developed in Appendix A. This modification is closer to the one discussed in [11], and in general terms is based in embedding the unknown inhomogeneity inside an artificially introduced inhomogeneity. Here we choose the artificial inhomogeneity with constitutive material properties of negative values which corresponds to metamaterials. We show that the resulting eigenvalue problem for this choice has a structure that resembles the Steklov eigenvalue problem discussed in Section 2 , but it provides richer spectral information.

In a similar way as in Section 2 , letting the bounded region $D_{b} \subset \mathbb{R}^{m}$ with smooth boundary $\partial D_{b}$ and a connected complement in $\mathbb{R}^{m}$ be such that $D \subseteq D_{b}$, we introduce the scattering problem

$$
\begin{array}{ll}
\Delta u_{b}^{s}+k^{2} u_{b}^{s}=0 & \text { in } \mathbb{R}^{m} \backslash \overline{D_{b}} \\
(-a) \Delta u_{b}+k^{2} \lambda u_{b}=0 & \text { in } D_{b} \\
u_{b}-u_{b}^{s}=u^{i} & \text { on } \partial D_{b} \\
(-a) \frac{\partial u_{b}}{\partial \nu}-\frac{\partial u_{b}^{s}}{\partial \nu}=\frac{\partial u^{i}}{\partial \nu} & \text { on } \partial D_{b} \\
\lim _{r \rightarrow \infty} r^{\frac{m-1}{2}}\left(\frac{\partial u_{b}^{s}}{\partial r}-i k u_{b}^{s}\right)=0 &
\end{array}
$$


where $u^{i}:=e^{i k x \cdot d}$ and $a>0$ is a fixed parameter such that $a \neq 1$ whereas $\lambda \in \mathbb{C}$. The scattering problem (42) is well-posed as long as $\Im(\lambda) \geq 0$ [4] (this models the scattering problem for the inhomogeneity with support $D_{b}$ with negative material properties, i.e. the so-called metamaterial). If $u_{b}^{\infty}(\hat{x}, d)$ denotes the far field pattern of $u_{b}^{s}$, the corresponding far field operator is given by

$$
\left(F_{b}^{\lambda} g\right)(\hat{x}):=\int_{S} u_{b}^{\infty}(\hat{x}, d) g(d) d s(d) .
$$

Note that $F_{b}^{\lambda} g:=u_{b, g}^{\infty}$ is the far field pattern of the radiating solution $u_{b, g}^{s}$ solving (42) with incident wave $u^{i}:=v_{b}$ being the Herglotz wave function with kernel $g$.

Similarly to the far field operator $F$ corresponding to the physical inhomogeneity discussed in Introduction, the far field operator $F_{b}^{\lambda}$ corresponding to the artificially induced background can be factorized as

$$
F_{b}^{\lambda} g=H_{b}^{*} T_{b} H_{b}
$$

Here $T_{b}: L^{2}(D) \times L^{2}(D) \rightarrow L^{2}(D) \times L^{2}(D)$ is defined by

$$
T_{b}(\varphi, \psi):=\gamma_{m}\left((1+a)\left(\varphi+\nabla w_{b}^{s}\right), k^{2}(\lambda-1)\left(\psi+w_{b}^{s}\right)\right)
$$

where $w_{b}^{s} \in H_{l o c}^{1}\left(\mathbb{R}^{3}\right)$ is the unique radiating solution of

$$
\tilde{a} \Delta w_{b}^{s}+k^{2} \tilde{\lambda} w_{b}^{s}=\nabla \cdot(1-\tilde{a}) \nabla \varphi+k^{2}(1-\tilde{\lambda}) \psi \text { in } \mathbb{R}^{m}
$$

with $(\tilde{a}, \tilde{\lambda})=(-a, \lambda)$ in $D_{b}$ and $(\tilde{a}, \tilde{\lambda})=(1,1)$ in $\mathbb{R}^{m} \backslash \overline{D_{b}}$, whereas $H_{b}: L^{2}(S) \rightarrow$ $L^{2}\left(D_{b}\right) \times L^{2}\left(D_{b}\right)$ and its $L^{2}$-adjoint $H_{b}^{*}: L^{2}\left(D_{b}\right) \times L^{2}\left(D_{b}\right) \rightarrow L^{2}(S)$ are defined by $(8)$ and (9), respectively, where $D$ is replaced by $D_{b}$.

We again define the corresponding modified far field operator $\mathcal{F}: L^{2}(S) \rightarrow L^{2}(S)$

$$
\mathcal{F} g:=F g-F_{b}^{\lambda} g \text {. }
$$

The modified far field operator $\mathcal{F} g$ can be seen as the far field pattern corresponding to the inhomogeneity $(A, n, D)$ due to incident field $u^{i}:=v_{g}-u_{b, g}^{s}$ where $u_{b, g}^{s}$ solves (42) with $u^{i}:=v_{g}$. This is saying that $\mathcal{F}$ corresponds to the scattering by the given inhomogeneity sitting in a new background obtained by subtracting from the physical homogeneous background the artificial metamaterial $\left(-a, \lambda, D_{b}\right)$.

To see what is the eigenvalue problem that arises in connection to $\mathcal{F}$, we again look at its injectivity. To this end, if $\mathcal{F} g=0$ then from Rellich's lemma and unique continuation argument we have that $u_{g}=u_{b, g}$ in $\mathbb{R}^{m} \backslash D_{b}$ (see (3) and (43)). Thus, extending $A=I$ and $n=1$ in $D_{b} \backslash \bar{D}$, and using the continuity of the Cauchy data of both total fields $u_{g}$ and $u_{b, g}$ across $\partial D_{b}$, we obtain that $v:=\left.u_{b, g}\right|_{D_{b}}$ and $w:=\left.u_{g}\right|_{D_{b}}$ satisfy the following set of homogenous equations

$$
\begin{array}{ll}
\nabla \cdot A \nabla w+k^{2} n w=0 & \text { in } D_{b} \\
(-a) \Delta v+k^{2} \lambda v=0 & \text { in } D_{b} \\
w=v & \text { on } \partial D_{b} \\
\frac{\partial w}{\partial \nu_{A}}=-a \frac{\partial v}{\partial \nu} & \text { on } \partial D_{b} .
\end{array}
$$


Thus, arguing in the same way as for the Steklov eigenvalues, the operator $\mathcal{F}$ is injective if (47) has only trivial solution. The values of $\lambda \in \mathbb{C}$ for which (47) has nonzero solutions $v \in H^{1}\left(D_{b}\right)$ and $w \in H^{1}\left(D_{b}\right)$ are the eigenvalues associated with this modified operator (in [11] these eigenvalues are referred to as modified transmission eigenvalues). Note that here $\lambda$ is the eigenvalue parameter and $k$ is fixed).

\subsection{Analysis of the New Eigenvalue Problem}

To study the eigenvalue problem (47), we first write it in the following equivalent variational form,

$$
\int_{D_{b}} A \nabla w \cdot \nabla \bar{w}^{\prime} d x+a \int_{D_{b}} \nabla v \cdot \nabla \bar{v}^{\prime} d x-k^{2} \int_{D_{b}} n w \bar{w}^{\prime} d x=-k^{2} \lambda \int_{D_{b}} v \bar{v}^{\prime} d x(48)
$$

for $\left(w^{\prime}, v^{\prime}\right) \in \mathcal{H}\left(D_{b}\right)$ where

$$
\mathcal{H}\left(D_{b}\right)=\left\{(w, v) \in H^{1}\left(D_{b}\right) \times H^{1}\left(D_{b}\right) \text { such that } w=v \text { on } \partial D_{b}\right\} .
$$

Obviously, if $\Im(A)=0$ and $\Im(n)=0$, the eigenvalues $\lambda$ are all real. In fact, for real valued coefficients $A$ and $n$, this is an eigenvalue problem for a compact selfadjoint operator. To see this, one possibility is to fix a real $\beta$ such that $k$ is not a transmission eigenvalue of

$$
\begin{array}{ll}
\nabla \cdot A \nabla w+k^{2} n w=0 & \text { in } D_{b} \\
(-a) \Delta v+k^{2} \beta v=0 & \text { in } D_{b} \\
w=v & \text { on } \partial D_{b} \\
\frac{\partial w}{\partial \nu_{A}}=-a \frac{\partial v}{\partial \nu} & \text { on } \partial D_{b} .
\end{array}
$$

This means that the selfadjoint operator $\mathbb{A}: \mathcal{H}\left(D_{b}\right) \rightarrow \mathcal{H}\left(D_{b}\right)$ defined by the Riesz representation as

$$
\left(\mathbb{A}(w, v),\left(w^{\prime}, v^{\prime}\right)\right)_{\mathcal{H}\left(D_{b}\right)}=\int_{D_{b}}\left(A \nabla w \cdot \nabla w^{\prime}+a \nabla v \cdot \nabla v^{\prime} d x-k^{2} n w w^{\prime}+k^{2} \beta v v^{\prime}\right) d x
$$

fo all $\left(w^{\prime}, v^{\prime}\right) \in \mathcal{H}\left(D_{b}\right)$ is invertible. We remark that the operator $\mathbb{A}$ is of Fredholm type and depends analytically on $\beta$. Moreover, $\mathbb{A}$ is coercive for $k>0$ and $\beta=i \tau$ with $\tau>0$. This proves, by the analytic Fredholm theory, that for any fixed $k>0$ there exists $\beta$ real such that $\mathbb{A}$ is invertible. Now consider the operator $\mathbb{T}: L^{2}(D) \rightarrow L^{2}(D)$ mapping

$$
\mathbb{T}: f \in L^{2}(D) \mapsto v_{f} \in H^{1}\left(D_{b}\right) \text { where }\left(w_{f}, v_{f}\right)=\mathbb{A}^{-1}(0, f),
$$

which is compact and selfadjoint. Therefore our eigenvalue problem for $\lambda$ becomes

$$
\mathbb{T} v=-k^{2}(\lambda-\beta) v
$$

which is an eigenvalue problem for a selfadjoint compact operator. This implies in particular the existence of an infinite set of real eigenvalues $\lambda$ which, as we show in the next theorem, accumulate only at $-\infty$. 
Remark 4. We note that our eigenvalue problem (48) has a similar structure with the Steklov eigenvalue problem (18). We remark that (47) with a positive parameter instead of $(-a)$ has a different structure, and for the case of $A=I$ it is investigated in [11] where the existence of eigenvalues is also proven for complex valued $n$. In particular, provided that $k>0$ satisfies Assumption 1 we can define the interior Dirichlet-toNeuman operator $\mathcal{N}_{k, A, n}: H^{1 / 2}\left(\partial D_{b}\right) \rightarrow H^{-1 / 2}\left(\partial D_{b}\right)$ as $\mathcal{N}_{k, A, n}: \varphi \mapsto \frac{\partial w_{\varphi}}{\partial \nu_{A}}$, where $w_{\varphi}$ satisfies

$$
\nabla \cdot A \nabla w_{\varphi}+k^{2} n w_{\varphi}=0 \quad \text { in } D_{b} \quad \text { and } \quad w_{\varphi}=\varphi \quad \text { on } \partial D_{b} .
$$

Then (47) with eigenvalue parameter $\lambda$ becomes a Robin type eigenvalue problem for the $-\Delta$ with nonlocal boundary condition:

$$
\begin{aligned}
& a \Delta v-k^{2} \lambda v=0 \quad \text { in } D_{b} \\
& a \frac{\partial v}{\partial \nu}-\mathcal{N}_{k, A, n} v=0 \quad \text { on } \partial D_{b} .
\end{aligned}
$$

Theorem 5. For real valued $A$ and $n$ and a fixed $k>0$ there exists at least one positive eigenvalue of (47). If in addition $k>0$ satisfies Assumption 1, then there are at most finitely many positive eigenvalues.

Proof. Assume to the contrary that all eigenvalues $\lambda_{j} \leq 0$. This means that

$$
\int_{D_{b}} \nabla w \cdot A \nabla \bar{w} d x+a \int_{D_{b}} \nabla v \cdot \nabla \bar{v} d x-k^{2} \int_{D_{b}} n|w|^{2} d s \geq 0
$$

for all $(w, v) \in \mathcal{H}\left(D_{b}\right)$ since due to self-adjoiness all the eigenfunctions $(w, v)$ form a Riesz basis for $\mathcal{H}\left(D_{b}\right)$. Now taking $w=1$ and $v=1$ yields a contradiction which proves the first statement.

Next we assume by contradiction that there exists a sequence of positive eigenvalues $\lambda_{j}>0, j \in \mathbb{N}$ converging to $+\infty$ with eigenfunctions $\left(w_{j}, v_{j}\right) \in \mathcal{H}\left(D_{b}\right)$ normalized such that

$$
\left\|w_{j}\right\|_{H^{1}\left(D_{b}\right)}+\left\|v_{j}\right\|_{H^{1}\left(D_{b}\right)}=1
$$

Then from

$$
\left(A \nabla w_{j}, \nabla w_{j}\right)+a\left(\nabla v_{j}, \nabla v_{j}\right)-k^{2}\left(n w_{j}, w_{j}\right)=-k^{2} \lambda_{j}\left(v_{j}, v_{j}\right)
$$

since the left hand side is bounded we obtain that $v_{j} \rightarrow 0$ in the $L^{2}\left(D_{b}\right)$. Next, up to a subsequence, $w_{j} \rightarrow w$ weakly in $H^{1}\left(D_{b}\right)$ and this weak limit satisfies $\nabla \cdot A \nabla w+k^{2} n w=0$ in $D_{b}$ and $w=0$ on $\partial D_{b}$. Our assumption on $k$ implies that $w=0$, i.e. $w_{j} \rightarrow 0$ weakly in $H^{1}\left(D_{b}\right)$ and up to a subsequence $w_{j} \rightarrow 0$ strongly in $L^{2}\left(D_{b}\right)$. From (53)

$$
\left(A \nabla w_{j}, \nabla w_{j}\right)+a\left(\nabla v_{j}, \nabla v_{j}\right) \leq k^{2}\left(n w_{j}, w_{j}\right), \quad \text { for all } j \in \mathbb{N} .
$$

Since $\left(n w_{j}, w_{j}\right) \rightarrow 0$, we conclude that

$$
\left(A \nabla w_{j}, \nabla w_{j}\right) \rightarrow 0, \quad \text { and } \quad a\left(\nabla v_{j}, \nabla v_{j}\right) \rightarrow 0 \quad \text { as } j \rightarrow \infty,
$$


which implies that $\left\|\nabla w_{j}\right\|_{H^{1}\left(D_{b}\right)} \rightarrow 0,\left\|\nabla v_{j}\right\|_{H^{1}\left(D_{b}\right)} \rightarrow 0$. This contradicts (52) and the proof of the theorem is completed.

For $(w, v) \in \mathcal{H}\left(D_{b}\right)$, since $w-v \in H_{0}^{1}\left(D_{b}\right)$ the Poincaré inequality holds

$$
\|w-v\|^{2} \leq C_{p}\|\nabla w-\nabla v\|^{2}
$$

with the optimal constant $C_{p}>0$ being the first Dirichlet eigenvalue for $-\Delta$ in $D_{b}$. Thus

$$
(w, w) \leq C_{p}(\nabla w, \nabla w)+C_{p}(\nabla v, \nabla v)+(v, v)
$$

In a similar manner as for the Steklov eigenvalue problem discussed in Section (2), we would like to find a $\Lambda>0$ such that

$$
\begin{aligned}
& \int_{D_{b}} A \nabla w \cdot \nabla \bar{w} d x+a \int_{D_{b}} \nabla v \cdot \nabla \bar{v} d x-k^{2} \int_{D_{b}} n|w|^{2} d x+\Lambda \int_{D_{b}}|v|^{2} d x \\
& \geq C\left(\|w\|_{H^{1}\left(D_{b}\right)}^{2}+\|v\|_{H^{1}\left(D_{b}\right)}^{2}\right)
\end{aligned}
$$

Obviously from (54), the coercivity (55) holds if $k^{2}<\frac{a_{\min }}{C_{p} n_{\max }}$ and $a$ is chosen large enough. In this case, our eigenvalue problem

$$
\begin{aligned}
\int_{D_{b}} A \nabla w \cdot \nabla \bar{w}^{\prime} d x+a & \int_{D_{b}} \nabla v \cdot \nabla \bar{v}^{\prime} d x-k^{2} \int_{D_{b}} n w \bar{w}^{\prime} d x \\
& +\Lambda \int_{D_{b}} v \bar{v}^{\prime} d x=-k^{2}(\lambda+\Lambda) \int_{D_{b}} v \bar{v}^{\prime} d x
\end{aligned}
$$

becomes a generalized eigenvalue problem for a positive compact selfadjoint operator and the eigenvalues $-\left(\lambda_{j}+\Lambda\right)$ satisfies Courant-Fischer min-max principle. Consequently we obtain that our largest positive eigenvalue $\lambda_{1}:=\lambda_{1}(A, n, k)$ satisfies

$$
\lambda_{1}=\inf _{(w, v) \in \mathcal{H}\left(D_{b}\right), v \neq 0} \frac{k^{2} \int_{D_{b}} n|w|^{2} d x-\int_{D_{b}} \nabla w \cdot A \nabla w d x-a \int_{D_{b}}|\nabla v|^{2} d x}{\int_{D_{b}}|v|^{2} d x} .
$$

Hence $\lambda_{1}$ depends monotonically increasing with respect $n$ and monotonically decreasing with respect to $A$. The above condition on $k^{2}$ for which (55) is satisfied can be improved. In the following theorem we obtain the same condition on $k$ as for the Steklov eigenvalues in Theorem 2.

Theorem 6. Assume that $k^{2}<\eta_{0}\left(A, n, D_{b}\right)$, where $\eta_{0}\left(A, n, D_{b}\right)$ is the first Dirichlet eigenvalue of (20). Then there is a $\Lambda>0$ such that (55) holds. In particular, in this case the largest positive eigenvalue satisfies (57).

Proof. Fix $k^{2}<\eta_{0}\left(A, n, D_{b}\right)$ and assume to the contrary that there exists a sequence of positive constants $\Lambda_{j}=j, j \in \mathbb{N}$, and a sequence of functions $\left(w_{j}, v_{j}\right) \in \mathcal{H}\left(D_{b}\right)$ normalized as $\left\|w_{j}\right\|_{H^{1}\left(D_{b}\right)}+\left\|v_{j}\right\|_{H^{1}\left(D_{b}\right)}=1$ such that

$$
\int_{D_{b}} \nabla w_{j} \cdot A \nabla \bar{w}_{j} d x+a \int_{D_{b}}\left|\nabla v_{j}\right|^{2} d x-k^{2} \int_{D_{b}} n\left|w_{j}\right|^{2} d x+j \int_{D_{b}}\left|v_{j}\right|^{2} d s \leq 0 .(58)
$$


From

$$
\int_{D_{b}} \nabla w_{j} \cdot A \nabla \bar{w}_{j} d x+a \int_{D_{b}}\left|\nabla v_{j}\right|^{2} d+j \int_{D_{b}}\left|v_{j}\right|^{2} d s \leq k^{2} \int_{D_{b}} n\left|w_{j}\right|^{2} d x
$$

we see that $j \int_{D_{b}}\left|v_{j}\right|^{2} d s$ is bounded which implies that $v_{j} \rightarrow 0$ strongly in $L^{2}\left(D_{b}\right)$. On the other hand, the boundedness implies that, up to a subsequence, $w_{j} \rightarrow w$ and $v_{j} \rightarrow 0$ weakly in $H^{1}\left(D_{b}\right)$. Since $\left(w_{j}, v_{j}\right) \in \mathcal{H}\left(D_{b}\right)$ we get in particular that $w \in H_{0}^{1}\left(D_{b}\right)$. By going to a subsequence, one can also assume that $w_{j} \rightarrow w$ strongly in $L^{2}\left(D_{b}\right)$. Since the norm of the weak limit is smaller that the lim-inf of the norm

$$
(A \nabla w, \nabla w) \leq \liminf _{j \rightarrow \infty} \int_{D_{b}} \nabla w_{j} \cdot A \nabla \bar{w}_{j} d x \leq \lim _{j \rightarrow \infty} k^{2} \int_{D_{b}} n\left|w_{j}\right|^{2} d x=k^{2}(n w, w)
$$

which contradicts the fact that

$$
k^{2}<\inf _{w \in H_{0}^{1}\left(D_{b}\right), w \neq 0} \frac{(A \nabla w, w)}{(n w, w)}=\eta_{1}\left(A, n, D_{b}\right) .
$$

This ends the proof.

\subsection{Determination of the New Eigenvalues from Far Field Data}

We end this section by showing how to determine the eigenvalues $\lambda$ of (47) from a knowledge of the modified far field operator (46) applying the generalized linear sampling method framework developed in Appendix A. The approach follows the line of the one developed for the Steklov eigenvalues, and therefore we shall only give a sketch of the proofs. To this end, the modified far field operator can be factorized as $\mathcal{F}=\mathcal{G H}$ where here $\mathcal{H}: L^{2}(S) \rightarrow L^{2}\left(D_{b}\right)^{m} \times L^{2}\left(D_{b}\right)$ is defined by

$$
\mathcal{H} g=\left(\left.\nabla u_{b, g}\right|_{D_{b}},\left.u_{b, g}\right|_{D_{b}}\right)
$$

with $u_{b, g}$ being the solution of (42) with $u^{i}=v_{g}$, whereas $\mathcal{G}: \overline{\mathcal{R}(\mathcal{H})} \subset L^{2}\left(D_{b}\right)^{m} \times$ $L^{2}\left(D_{b}\right) \rightarrow L^{2}(S)$ is defined by

$$
\mathcal{G}(\varphi, \psi)=w^{\infty}
$$

with $w^{\infty}$ being the far field of $w^{s} \in H_{l o c}^{1}\left(\mathbb{R}^{m}\right)$ that solves

$$
\nabla \cdot A \nabla w^{s}+k^{2} n w^{s}=\nabla \cdot(-a-A) \varphi+k^{2}(\lambda-n) \psi \quad \text { in } \mathbb{R}^{m}
$$

together with the Sommerfeld radiation condition, and $\overline{\mathcal{R}(\mathcal{H})}$ is the closure of the range of $\mathcal{H}$ in $L^{2}\left(D_{b}\right) \times L^{2}\left(D_{b}\right)$.

Similarly to Section 3, we shall apply Theorem 8 in Appendix A to $\mathcal{F}$ with $X=X^{*}:=$ $L^{2}(S)$ and $Y:=L^{2}\left(D_{b}\right)^{m} \times L^{2}\left(D_{b}\right)$. We here discuss only the case $B(g)=\left|\left(F_{b}^{\lambda} g, g\right)\right|$.

Lemma 4. Let $F_{b}^{\lambda}$ be defined by (43). Then the operator $B: L^{2}(S) \rightarrow \mathbb{R}_{+}$defined by $B(g):=\left|\left(F_{b}^{\lambda} g, g\right)\right|$ satisfies Assumption 3 in Appendix $A$ with $H:=\mathcal{H}$ if $D \subseteq D_{b}$ and 
$k, \lambda$ and $a$ are such that

$$
\begin{array}{ll}
\Delta w+k^{2} w=0 & \text { in } D_{b} \\
(-a) \Delta v+k^{2} \lambda v=0 & \text { in } D_{b} \\
w=v & \text { on } \partial D_{b} \\
\frac{\partial w}{\partial \nu}=-a \frac{\partial v}{\partial \nu} & \text { on } \partial D_{b}
\end{array}
$$

has only the trivial solution in $\mathcal{H}\left(D_{b}\right)$.

Proof. The assumption stated in the lemma guaranties that $T_{b}$ defined by (45) is coercive (see e.g Theorem 2.42 in [5]). Then the proof follows exactly the lines of the proof of the first part of Lemma 1 using factorization (44).

Note that the assumption on the uniqueness of solutions of (63) is a natural assumption since it means in particular that $\lambda$ should not be also an eigenvalue for the case $A=I$ and $n=1$. It is indeed possible to play with the parameter $a$ to enforce this assumption to be true for all eigenvalues $\lambda$. If this assumption fails for all eigenvalues $\lambda$ then this simply means that the set of these eigenvalues does not differentiate the inhomogeneity from the vacuum: in other words the inhomogeneity is invisible to the considered spectrum. Studying this inverse spectral question has its own interest and can be an interesting future work.

We now proceed with the following two lemmas which allow to derive a characterization of the eigenvalues $\lambda$ form scattering data.

Lemma 5. Assume that $\lambda$ is not an eigenvalue of (47). Then $\Phi_{z}^{\infty} \in \mathcal{R}(\mathcal{G})$ for $z \in D_{b}$.

Proof. Following the same argument as in the proof of Lemma 2.38 in [5], we first observe that $(\varphi, \psi) \in \overline{\mathcal{R}(\mathcal{H})}$ if and only if $\varphi:=\nabla u_{b}$ and $\psi=u_{b}$ where $u_{b} \in H^{1}\left(D_{b}\right)$ and satisfies

$$
(-a) \Delta u_{b}+k^{2} \lambda u_{b}=0 \quad \text { in } D_{b}
$$

Fix a $z \in D_{b}$ and let $w_{z}$ and $v_{z}$ in $H^{1}\left(D_{b}\right)$ be the unique solution of

$$
\begin{array}{ll}
\nabla \cdot A \nabla w_{z}+k^{2} n w_{z}=0 & \text { in } D_{b} \\
(-a) \Delta v_{z}+k^{2} \lambda v_{z}=0 & \text { in } D_{b} \\
w_{z}-v_{z}=\Phi(\cdot, z) & \text { on } \partial D_{b} \\
\frac{\partial w_{z}}{\partial \nu_{A}}+a \frac{\partial v_{z}}{\partial \nu}=\frac{\partial \Phi(\cdot, z)}{\partial \nu} & \text { on } \partial D_{b} .
\end{array}
$$

We extend $w_{z}^{s}:=w_{z}-v_{z}$ by $\Phi(\cdot, z)$ outside $D_{b}$. Then obviously, $w_{z}^{s} \in H_{l o c}^{1}\left(\mathbb{R}^{m}\right)$ and satisfies (62) with $\varphi_{z}:=\nabla v_{z}$ and $\psi_{z}=v_{z}$. We then conclude that $\left(\varphi_{z}, \psi_{z}\right) \in \mathcal{R}(\mathcal{H})$ and by construction $\mathcal{G}\left(\varphi_{z}, \psi_{z}\right)=\Phi_{z}^{\infty}$.

Lemma 6. Assume that $\lambda$ is an eigenvalue of (47) and $\lambda$ is not an eigenvalue of (63). Then the set of points z such that $\Phi_{z}^{\infty} \in \mathcal{R}(\mathcal{G})$ is nowhere dense in $D_{b}$. 
Proof. The proof is similar to Theorem 3.3 in [7]. Assume to the contrary that $\mathcal{G}\left(\varphi_{z}, \psi_{z}\right)=\Phi_{z}$ for $z$ is a dense subset of a ball $B \subset D_{b}$. By definition (61) we have that $\left.\nabla v_{z}\right|_{D_{b}}:=\varphi_{z}$ and $\left.v_{z}\right|_{D_{b}}:=\psi_{z}$ and $(-a) \Delta v_{z}+k^{2} \lambda v_{z}=0$ in $D_{b}$. Using Rellich lemma we conclude that these $v_{z}$ and $w_{z}$ in the definition $(61)$ of $\mathcal{G}\left(\varphi_{z}, \psi_{z}\right)$ satisfy

$$
\begin{array}{ll}
\nabla \cdot A \nabla w_{z}+k^{2} n w_{z}=0 & \text { in } D_{b} \\
(-a) \Delta v_{z}+k^{2} \lambda v_{z}=0 & \text { in } D_{b} \\
w_{z}-v_{z}=\Phi(\cdot, z) & \text { on } \partial D_{b} \\
\frac{\partial w_{z}}{\partial \nu_{A}}+a \frac{\partial v_{z}}{\partial \nu}=\frac{\partial \Phi(\cdot, z)}{\partial \nu} & \text { on } \partial D_{b} .
\end{array}
$$

Let $\left(w_{\lambda}, v_{\lambda}\right)$ be an eigenpair associated with $\lambda$. Multiplying the equation for $w_{z}$ in by $w_{\lambda}$ and applying the Green formula twice implies that

$$
\int_{\partial D_{b}}\left(\frac{\partial w_{z}}{\partial \nu_{A}} w_{\lambda}-w_{z} \frac{\partial w_{\lambda}}{\partial \nu_{A}}\right) d s=0
$$

Similarly

$$
-a \int_{\partial D_{b}}\left(\frac{\partial v_{z}}{\partial \nu} v_{\lambda}-v_{z} \frac{\partial v_{\lambda}}{\partial \nu}\right) d s=0 .
$$

Adding the two equations and using the boundary conditions we obtain

$$
\begin{aligned}
0 & =\int_{\partial D_{b}}\left(\frac{\partial \Phi(\cdot, z)}{\partial \nu} \bar{w}_{\lambda}-\Phi(\cdot, z) \frac{\partial \bar{w}_{\lambda}}{\partial \nu_{A}}\right) d s \\
& =\int_{\partial D_{b}}\left(\frac{\partial \Phi(\cdot, z)}{\partial \nu} \bar{v}_{\lambda}-(-a) \Phi(\cdot, z) \frac{\partial \bar{v}_{\lambda}}{\partial \nu}\right) d s
\end{aligned}
$$

This implies in particular that (the incident field)

$$
v_{\lambda}^{i}(z):=\int_{\partial D_{b}}\left(\frac{\partial \Phi(\cdot, z)}{\partial \nu} \bar{v}_{\lambda}-(-a) \Phi(\cdot, z) \frac{\partial \bar{v}_{\lambda}}{\partial \nu}\right) d s=0
$$

for $z$ is a dense subset of a ball $B \subset D_{b}$ and, by analyticity in all of $D_{b}$. Next, let us define

$$
v_{\lambda}^{s}:=v_{\lambda}-v_{\lambda}^{i} \text { in } D_{b}
$$

and

$$
v_{\lambda}^{s}(x):=\int_{\partial D_{b}}\left(\frac{\partial \Phi(\cdot, x)}{\partial \nu} v_{\lambda}+a \Phi(\cdot, x) \frac{\partial v_{\lambda}}{\partial \nu}\right) d s \quad x \in \mathbb{R}^{m} \backslash \overline{D_{b}}
$$

Then $v_{\lambda}^{s}$ is a solution of (42) with $u^{i}=0$. Therefore $v_{\lambda}^{s}=0$ and then $v_{\lambda}=0$ in $D_{b}$. Similar arguments also show that $w_{\lambda}=0$, which gives a contradiction.

Finally we are ready to apply Theorem 8 in Appendix A to the operator $\mathcal{F}$ using Lemma 5 and Lemma 6. 
Eigenvalues in Inverse Scattering Theory

Theorem 7. Let $\lambda \in \mathbb{C}$ and assume that the modified far field operator $\mathcal{F}: L^{2}(S) \rightarrow$ $L^{2}(S)$ has dense range and that the assumptions of Lemma 4 are verified. Consider the functional

$$
J_{\alpha}\left(\Phi_{z}^{\infty}, g\right):=\alpha\left|\left(F_{b}^{\lambda} g, g\right)\right|+\left\|\mathcal{F} g-\Phi_{z}^{\infty}\right\|^{2} \quad \text { and set } \quad j_{\alpha}\left(\Phi_{z}^{\infty}\right):=\inf _{g} J_{\alpha}\left(\Phi_{z}^{\infty}, g .\right)
$$

Let $g_{\alpha}^{z}$ be a minimizing sequence defined by

$$
J_{\alpha}\left(\Phi_{z}^{\infty}, g_{\alpha}^{z}\right) \leq j_{\alpha}\left(\Phi_{z}^{\infty}\right)+C \alpha
$$

where $C>0$ is fixed. Then $\lambda$ is an eigenvalue of (47) if and only if the set of points $z$ for which $\left|\left(F_{b}^{\lambda} g_{\alpha}^{z}, g_{\alpha}^{z}\right)\right|$ is bounded as $\alpha \rightarrow 0$ is nowhere dense in $D_{b}$.

For the case of noisy data see the remarks at the end of Section 3.

\subsection{Numerical Examples}

To illustrate the viability of our method for determining the eigenvalues $\lambda$ from the modified far field operator, we present first some simple numerical examples for the case of a two-dimensional radially symmetric and isotropic inhomogeneity with real constant coefficients $A$ and $n$. We shall consider only the case of the new set of eigenvalues introduced in Section 4.1. To this end we assume that $D_{b}:=B_{R}$ is a ball of radius $R$ and consider the case when $D=D_{b}$. Then the fields that solve (47) for a fixed constant $a>0$ and $\lambda \in \mathbb{R}$ (note that in this case of eigenvalues $\lambda$ are real in cylindrical coordinates $(r, \theta)$ for $r \leq R$ can be written as:

$$
w(r, \theta)=\sum_{m=-\infty}^{+\infty} b_{m} J_{m}\left(k \sqrt{\frac{n}{A}} r\right) e^{i m \theta}, \quad v(r, \theta)=\sum_{m=-\infty}^{+\infty} c_{m} J_{m}\left(k \sqrt{\frac{\lambda}{-a}} r\right) e^{i m \theta}
$$

where $J_{m}$ are the Bessel functions of order $m$ and the coefficients $b_{m}$ and $c_{m}$ are real. Then $\lambda$ is an eigenvalue of (47) if and only if for some $m$

$$
\operatorname{det}\left(\begin{array}{cl}
J_{m}\left(k \sqrt{\frac{\lambda}{-a}} R\right) & -J_{m}\left(k \sqrt{\frac{n}{A}} R\right) \\
-a k \sqrt{\frac{\lambda}{-a}} J_{m}^{\prime}\left(k \sqrt{\frac{\lambda}{-a}} R\right) & -A k \sqrt{\frac{n}{A}} J_{m}^{\prime}\left(k \sqrt{\frac{n}{A}} R\right)
\end{array}\right)=0 .
$$

The zeros of this determinant will provide us with the eigenvalues of interest which we

will compare to the ones given using the characterization of Theorem 7 . Thanks to the symmetry of the problem, the far field pattern due to a Herglotz function with density

$$
g(\theta)=\sum_{-\infty}^{+\infty} a_{n} e^{i n \theta}
$$

as incident field, takes the form

$$
\begin{aligned}
u^{\infty}(\phi, \theta) & =\sum_{m=-\infty}^{+\infty} \frac{1}{d_{m}}\left[-A k \sqrt{\frac{n}{A}} J_{m}^{\prime}\left(k \sqrt{\frac{n}{A}} R\right) J_{m}(k R)\right. \\
& \left.+k J_{m}\left(k \sqrt{\frac{n}{A}} R\right) J_{m}^{\prime}(k R)\right] 2 \pi i^{m} a_{m} e^{i m \phi}
\end{aligned}
$$


where $d_{m}$ is given by

$$
d_{m}=\operatorname{det}\left(\begin{array}{cc}
J_{m}\left(k \sqrt{\frac{n}{A}} R\right) & -H_{m}^{(1)}(k R) \\
A k \sqrt{\frac{n}{A}} J_{m}^{\prime}\left(k \sqrt{\frac{n}{A}} R\right) & -k H_{m}^{(1)^{\prime}}(k R)
\end{array}\right) .
$$

This formula provide us now with an analytic expression of the far field operator $F$. A similar formula holds for $F_{b}^{\lambda}$ if we substitute $n$ with $\lambda$ and $A$ with $-a$. In order to ease the analytic expressions involved, we modify the penalty term in the cost functional $J_{\alpha}$ by considering

$$
\mathbb{J}_{\alpha}\left(\Phi_{z}^{\infty}, g\right)=\alpha\left\|\left(F_{b}^{\lambda *} F_{b}^{\lambda}\right)^{1 / 4} g\right\|^{2}+\left\|\mathcal{F} g-\Phi_{z}^{\infty}\right\|^{2}
$$

instead of the one in Theorem 7. As explained in $[2,1]$ (see also [5, Section 2.5]) the use of this penalty term for the general linear sampling method is possible as long as the operator is normal, which is the case when all the coefficients are real. It has the advantage of leading to a convex functional whose minimizer $g_{z}^{\lambda}$ can be computed in terms of the singular value decomposition. We shall then use

$$
I(\lambda):=\int_{D_{b}}\left\|\left(F_{b}^{\lambda *} F_{b}^{\lambda}\right)^{1 / 4} g_{z}^{\lambda}\right\|^{2} d z
$$

as an indicator function for the eigenvalues $\lambda$. This quantity is supposed to blow up at these values.

Taking advantage of the above analytic expressions for the far field operators one can also derive an analytic expression for $I(\lambda)$. To this end, one observes from (66) that $\phi \mapsto e^{i m \phi}$ are the singular vectors of both $F$ and $F_{b}^{\lambda}$ and the corresponding singular values for $F$ are given by

$$
\mu_{m}^{\infty}:=\left|\frac{1}{d_{m}}\left[-A k \sqrt{\frac{n}{A}} J_{m}^{\prime}\left(k \sqrt{\frac{n}{A}} R\right) J_{m}(k R)+k J_{m}\left(k \sqrt{\frac{n}{A}} R\right) J_{m}^{\prime}(k R)\right] 2 \pi\right| .
$$

The singular values $\mu_{m}^{b, \lambda \infty}$ of $F_{b}^{\lambda}$ have the same expression by substituting $A$ with $-a$ and $n$ with $\lambda$. Using the fact that

$$
\Phi_{z}^{\infty}=\sum_{m=-\infty}^{+\infty} i^{m}(-1)^{m} J_{m}(k|z|) e^{i m \phi}
$$

one can then get

$$
\left\|\left(F_{b}^{\lambda *} F_{b}^{\lambda}\right)^{1 / 4} g_{z}^{\lambda}\right\|^{2}=\sum_{m=-\infty}^{+\infty} \frac{\left(\mu_{m}^{\infty}-\mu_{m}^{b, \lambda \infty}\right)^{3}}{\left(\left(\mu_{m}^{\infty}-\mu_{m}^{b, \lambda \infty}\right)^{2}+\alpha \mu_{m}^{b, \lambda \infty}\right)^{2}} J_{m}(k|z|)^{2} 4 \pi^{2} .
$$

Integrating this quantity over $B_{R}$ then lead to (thanks to integral formula for Bessel functions)

$$
I(\lambda)=\sum_{m=-\infty}^{+\infty} \frac{\left(\mu_{m}^{\infty}-\mu_{m}^{b \infty}\right)^{3}}{\left(\left(\mu_{m}^{\infty}-\mu_{m}^{b \infty}\right)^{2}+\alpha \mu_{m}^{b \infty}\right)^{2}} 4 \pi^{3} R^{2}\left(J_{m}(k R)^{2}-J_{m-1}(k R) J_{m+1}(k R)\right) .
$$


In Figure 1 we show the results obtained for $I(\lambda)$ computed using the above analytic formula for the case when $(A, n,-a, k, R)=(2,8,-3,1,0.5)$ and $m \in[-100,100]$. We indeed observe peaks in the plot of $I(\lambda)$ in Figure 1, which coincides with the exact eigenvalues obtained using (65) (marked with red cross in the figure). The analytic

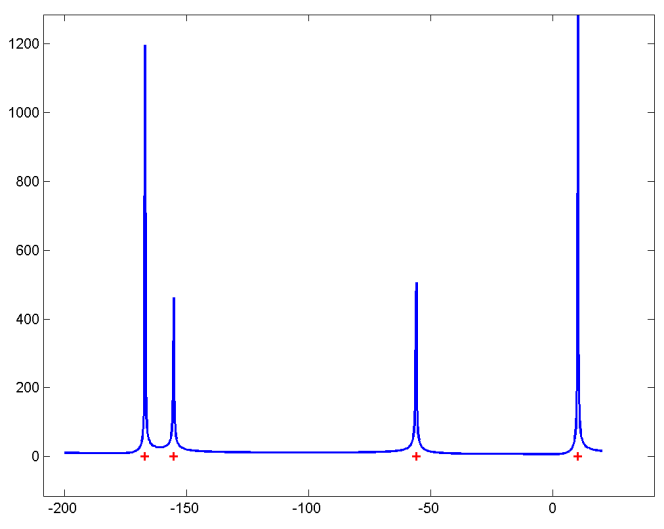

Figure 1. Plot of the analytic expression (67) of $I(\lambda)$ against $\lambda$ for $(A, n,-a, k, R)=$ $(2,8,-3,1,0.5)$ and $m \in[-100,100]$. The red crosses indicate the eigenvalues using the zeros of the determinant (65).

formula is fast to compute and therefore can be helpful in studying the dependence of the eigenvalues on the material properties of the inhomogeneity. Figure 2 shows the behavior of the indicator function with respect to $n, A$. This confirms in particular the monotonicity property indicated by the theory. One also observes that some eigenvalues may be much more sensitive than the others, making them a better candidate for obtaining information about the material properties.

In the spirit of using these eigenvalues for non destructive testing, we also derived an analytic formula for $I(\lambda)$ for the case of two layered media formed by two concentric ball $B_{R}$ and $B_{R_{0}}$ with $R_{0}<R$, where the coefficients $A_{0}$ and $n_{0}$ inside $B_{R_{0}}$ may be different from the coefficients $A$ and $n$ in $B_{R} \backslash B_{R_{0}}$. Figure 3 shows the behavior of the eigenvalues in terms of $R_{0}$. We also observe that different eigenvalues are not affected in the same way if we vary the radius of the inclusion. Of course more numerical investigation is needed to understand the relationship of the eigenvalues $\lambda$ with the material properties of the media. Furthermore, of interest is the understanding of the role of the artificial parameter $a$ in the sensitivity of the eigenvalues on the material properties $A$ and $n$. In the case of $-a>0$ and $A=1$ we refer the reader to the numerical examples presented in [11] for partial answer to these questions.

We now present some numerical results using numerical approximation of the modified far field operator $\mathcal{F}$. The numerical scheme for implementing the indicator function based on the generalized linear sampling method (GLSM)is the same as in [2]. To validate our numerical method, we first consider the case of $D_{b}=B_{R}$ as for the previous examples. Figure 4 shows the results for different percentage of additive 


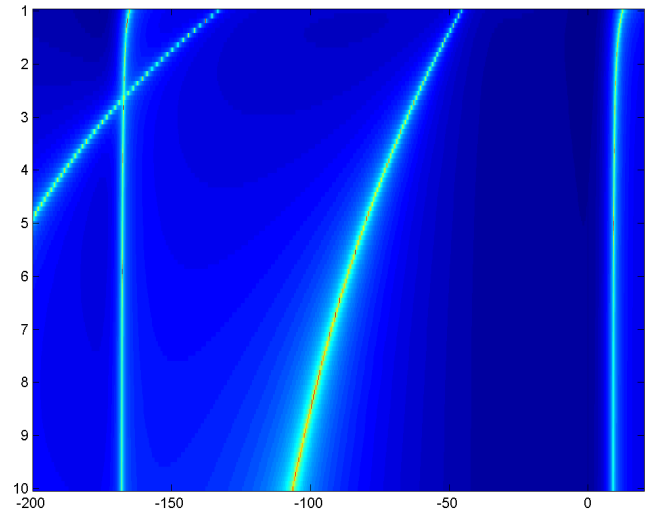

$(\lambda, A) \mapsto \log (I(\lambda))$

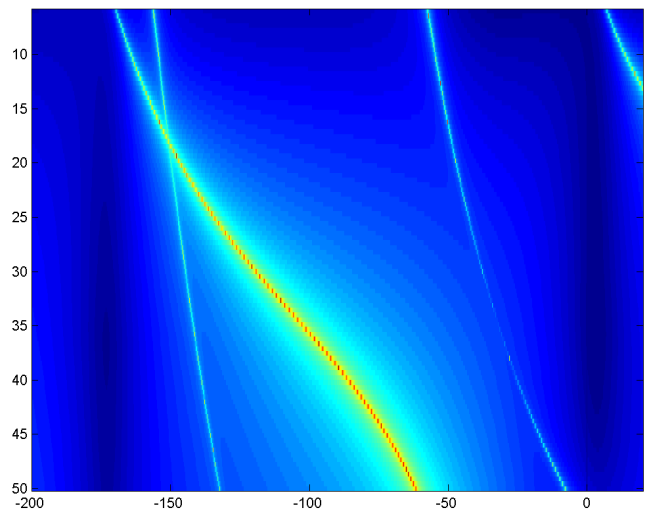

$(\lambda, n) \mapsto \log (I(\lambda))$

Figure 2. Left: plot of $\log (I(\lambda))$ in terms of $\lambda$ in abscissa and $A$ in ordiante varying from 1 to 10. Right: plot of $\log (I(\lambda))$ in terms of $\lambda$ in abscissa and $n$ varying from 5 to 50 . The bright color indicates large values of $I(\lambda)$. The non varying parameters are the same as in Figure 1

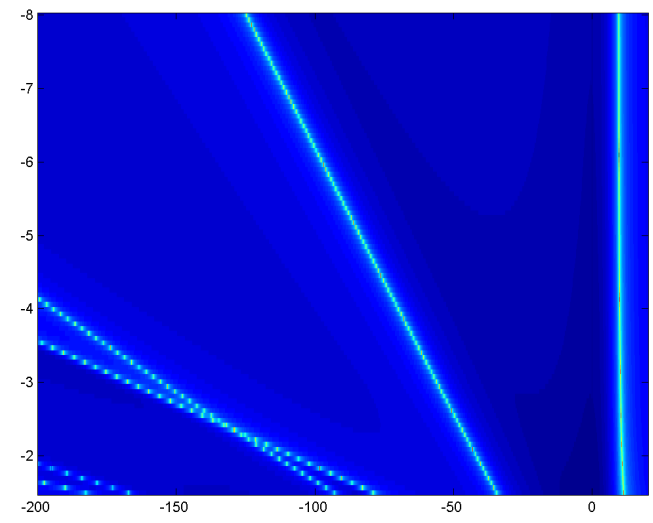

Figure 3. Plot of $\left(\lambda, R_{0}\right) \mapsto \log (I(\lambda))$ in terms of $\lambda$ in abscissa and $R_{0}$ in ordiante, varying from $2 \% R$ to $98 \% R$. The bright color indicates large values of $I(\lambda)$. $\left(A_{0}, n_{0}\right)=(2,15)$ and the other parameters are the same as in Figure 1

noise levels. We observe in particular that some eigenvalues (especially the largest positive) are robust with respect to the noise. Finally we consider an example for more general domain $D_{b}$ depicted 5 (left) with the same parameters as above, namely $(A, n,-a, k)=(2,8,-3,1)$. As explained in Section 4.1 for real valued $A, n$ the eigenvalue problem (47) is self ajoint, hence it is possible to solve it using classical finite element method. In particular we use Freefem ++ [15] to obtain a numerical approximation of these eigenvalues and compare them against the eigenvalues identified using the indicator function from the GLSM for $\lambda \in[-60,20]$. The results are presented in Figure 5 which confirms that our method works here as well as for the disk. 

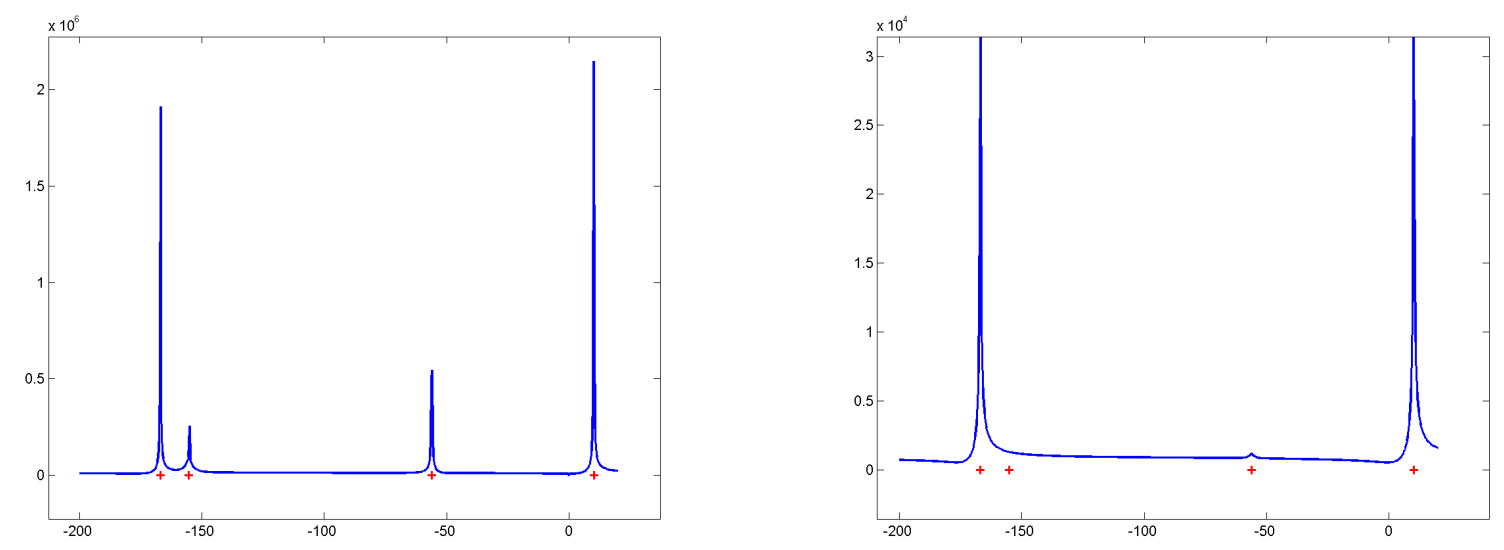

Figure 4. Plot of $I(\lambda)$ using the GLSM algorithm for the case $D_{b}=B_{R}$ and with $(A, n,-a, k, R)=(2,8,-3,1,0.5)$. Left: $1 \%$ added noise - Right $5 \%$ added noise. The red crosses indicate the eigenvalues using the zeros of the determinant (65).
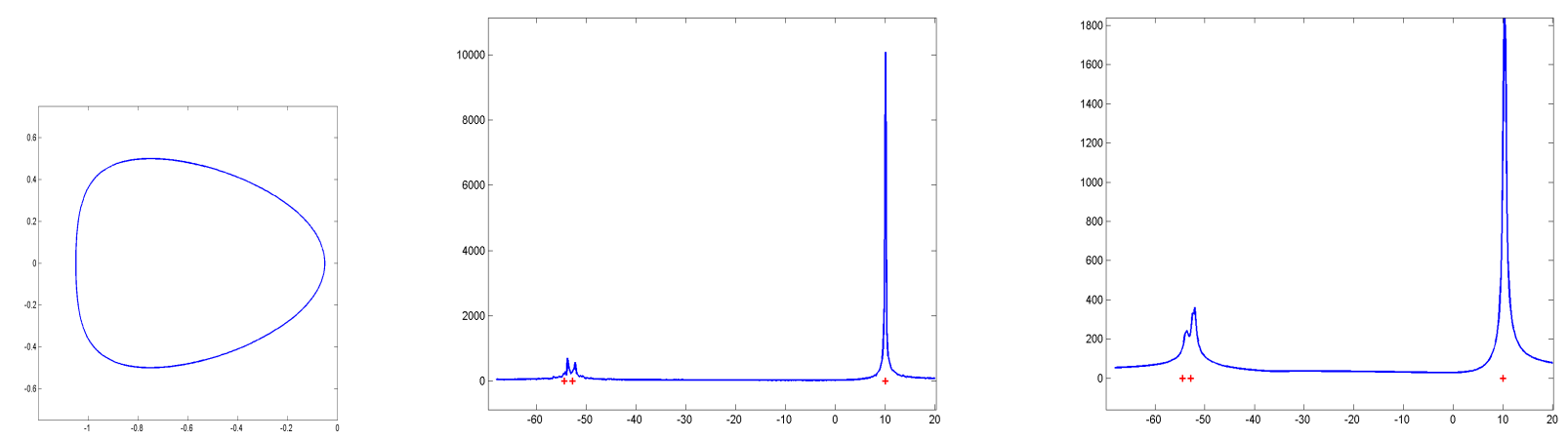

Figure 5. Plot of $I(\lambda)$ using the GLSM algorithm for $D_{b}$ being a kite depicted left and with $(A, n,-a, k)=(2,8,-3,1)$. Middle: $1 \%$ added noise - Right $5 \%$ added noise. The red crosses indicate the eigenvalues computed using FreeFem ++ for solving the eigenvalue problem (47).

\section{Appendix A. Analytical Framework for GLSM}

We develop here the abstract framework used for determining the interior eigenvalues. The main theorem below is a slight modification of the Generalized Linear Sampling Method (GLSM) introduced in [1] and [2] in order to address weaker assumptions on the penalty term.

Let $X$ and $Y$ be two complex reflexive Banach spaces with duals $X^{*}$ and $Y^{*}$. We consider a bounded linear operator $F: X \rightarrow X^{*}$ which assumes the factorization $F=G H$ where $H: X \rightarrow Y$ and $G: \overline{\mathcal{R}(H)} \subset Y \rightarrow X^{*}$ are bounded linear operators with $\overline{\mathcal{R}(H)}$ being the closure of the range of $H$ in $Y$. In addition let $B: X \rightarrow \mathbb{R}^{+}$be a continuous functional such that it satisfies the following fundamental assumption. 
Assumption 3. Given a sequence $\left\{g_{n}\right\} \in X$, the sequence $\left\{B\left(g_{n}\right)\right\}$ is bounded if and only if the sequence $\left\{\left\|H g_{n}\right\|_{Y}\right\}$ is bounded.

For a given parameter $\alpha>0$ and $\phi \in X^{*}$ we consider the following cost functional

$$
J_{\alpha}(g, \phi)=\alpha B(g)+\|F g-\phi\|^{2}
$$

This cost functional has no minimizer in general, however its positivity implies that we can define $j_{\alpha}(\phi):=\inf _{g \in X} J_{\alpha}(g, \phi)$.

The central theorem of the GLSM is the following characterization of the range of $G$ in terms $F$ and $B$. The proof of Theorem 8 is almost identical to the proof of Theorem 3 in [2] and we include here for readers convenience. A minor improvement in the proof below is the fact that $B$ does not need to satisfy a coercivity condition but only Assumption 3.

Theorem 8. In addition to Assumption 3 we assume that $F$ has dense range. Let $C>0$ be a given constant independent of $\alpha$ and consider a minimizing sequence $\left\{g_{\alpha}\right\}$ of $J_{\alpha}$, such that:

$$
J_{\alpha}\left(\phi, g_{\alpha}\right) \leq j_{\alpha}(\phi)+C \alpha
$$

Then $\phi \in \mathcal{R}(G)$ if and only if the sequence $B\left(g_{\alpha}\right)$ is bounded as $\alpha \rightarrow 0$.

Proof. Consider first the case $\phi \in \mathcal{R}(G)$. Then by definition we can find $\varphi \in \overline{\mathcal{R}(H)}$ such that $G \varphi=\phi$. Next, for a given but fixed $\alpha>0$, there exists $\tilde{g}_{\alpha} \in X$ such that $\left\|H \tilde{g}_{\alpha}-\varphi\right\|^{2}<\alpha$. Then by continuity of $G$, we can conclude that $\left\|F \tilde{g}_{\alpha}-\phi\right\|^{2}<\alpha\|G\|^{2}$. On the other hand, by Assumption 3, the sequence $B\left(\tilde{g}_{\alpha}\right)$ since bounded. Now the definition of $j_{\alpha}(\phi), g_{\alpha}$ and $J_{\alpha}$ yield

$$
\alpha B\left(g_{\alpha}\right) \leq J_{\alpha}\left(\phi, g_{\alpha}\right) \leq J_{\alpha}\left(\phi, \tilde{g}_{\alpha}\right)+C \alpha \leq C^{\prime} \alpha
$$

where $C^{\prime}$ is a constant independent of $\alpha$. Therefore the sequence $B\left(g_{\alpha}\right)$ is bounded as $\alpha \rightarrow 0$.

Now let us consider the case $\phi \notin \mathcal{R}(G)$ and assume to the contrary that $\lim _{\alpha \rightarrow 0} B\left(g_{\alpha}\right)<$ $+\infty$. Assumption 3 implies that $\left\|H g_{\alpha}\right\|$ is bounded independently from $\alpha$. Since $Y$ is reflexive one can extract a subsequence $H g_{\alpha}$ that weakly converge to some $\varphi$ in $Y$. We now observe that since $F$ has dense range then $j_{\alpha}(\phi) \rightarrow 0$ as $\alpha \rightarrow 0$ (see for instance Lemma 2 in [2]). Then, the definition of $J_{\alpha}\left(\phi, g_{\alpha}\right)$ implies that $F g_{\alpha}$ converges to $\phi$. On the other hand the fact that $F=G H$ and the uniqueness of the limit implies that $G \varphi=\phi$, which is a contradiction. We then conclude that $\lim _{\alpha \rightarrow 0} B\left(g_{\alpha}\right)=+\infty$.

\section{Acknowledgments}

The research of F. Cakoni is supported in part by AFOSR grant FA9550-17-1-0147, NSF Grant DMS-1602802 and Simons Foundation Award 392261. F. Cakoni gratefully acknowledges the financial support and hospitality of the INRIA DeFI Team during her visit at École Polytechnique when part of this work was completed. 


\section{References}

[1] L. Audibert, Qualitative Methods for Heterogeneous Media, PhD thesis, École Polytechnique, Palaiseau, France, 2015.

[2] L. Audibert and H. Haddar, A generalized formulation of the linear sampling method with exact characterization of targets in terms of far field measurements. Inverse Problems 30035011 (2014).

[3] L. Audibert and H. Haddar, The generalized linear sampling method for limited aperture measurements, SIAM J. Imaging Sci. 10 845-870, (2017).

[4] A.S. Bonnet-Ben Dhia, C. Carvalho, L. Chesnel, P. Ciarlet Jr, On the use of perfectly matched layers at corners for scattering problems with sign-changing coefficients. J. Comput. Phys. 322 (2016).

[5] F. Cakoni, D. Colton and H. Haddar, Inverse Scattering Theory and Transmission Eigenvalues, CBMS Series, SIAM Publications, 882016.

[6] F. Cakoni, D. Colton, S. Meng and P. Monk, Stekloff eigenvalues in inverse scattering SIAM J. Appl. Math. 76 1737-1763 (2016).

[7] F. Cakoni, D. Colton, and H. Haddar, On the determination of Dirichlet and transmission eigenvalues from far field data, C.R. Acad. Sci. Paris, Ser. 1348 379-383 (2010).

[8] F. Cakoni, D. Gintides and H. Haddar, The existence of an infinite discrete set of transmission eigenvalues. SIAM J. Math. Anal. 42 237-255 (2010).

[9] F. Cakoni, D. Colton, and P. Monk, On the use of transmission eigenvalues to estimate the index of refraction from far field data. Inverse Problems, 23 507-522 (2007).

[10] M. Chamaillard, N. Chaulet and H. Haddar, Analysis of the factorization method for a general class of boundary conditions. J. Inverse Ill-Posed Probl. 22 643-670 (2014)

[11] S. Cogar, D. Colton, S. Meng and P. Monk, Modified transmission eigenvalues in inverse scattering theory (to appear).

[12] D. Colton and R. Kress. Inverse Acoustic and Electromagnetic Scattering Theory. Springer, New York, 3nd Edition, 2013.

[13] G. Giorgi and H. Haddar, Computing estimates of material properties from transmission eigenvalues, Inverse Problems, 28 paper 055009 (2012).

[14] I. Harris, F. Cakoni and J. Sun, Transmission eigenvalues and non-destructive testing of anisotropic magnetic materials with voids. Inverse Problems, 30 paper 035016 (2014).

[15] F. Hecht, New development in FreeFem++, J. Numer. Math., 20(3-4), 251-265, (2012).

[16] A. Henrot, Extremum Problems for Eigenvalues of Elliptic Operators, Birkhauser Verlag, 2000.

[17] A. Kirsch and N. Grinberg The Factorization Method for Inverse Problems, Oxford University Press, Oxford, UK, 2008.

[18] A. Kirsch and A. Lechleiter, The inside-outside duality for scattering problems by inhomogeneous media. Inverse Problems 29104011 (2013).

[19] A. Kirsch and X. Liu, A modification of the factorization method for the classical acoustic inverse scattering problems. Inverse Problems 30035013 (2014).

[20] A. Lechleiter and S. Peters, Determining transmission eigenvalues of anisotropic inhomogeneous media from far field data. Commun. Math. Sci. 131803-1827 (2015).

[21] R.B. Melrose, Geometric Scattering Theory. Cambridge University Press, Cambridge, 1995.

[22] S. Peters and A. Kleefeld, Numerical computations of interior transmission eigenvalues for scattering objects with cavities, Inverse Problems 32045001 (2016).

[23] V. Petkov and L. Stoyanov, Geometry of the Generalized Geodesic Flow and Inverse Spectral Problems, Wiley, 2nd Edition, 2017.

[24] J. Sun and A. Zhou, Finite Element Methods for Eigenvalue Problems, CRC Press, 2016.

[25] G. Vodev, High-Frequency approximation of the interior Dirichlet-to-Neumann mao and applications to the transmission eigenvalues, arXiv:1701.04668v2. 